ARTICLE

https://doi.org/10.1038/s41467-019-11437-w

\title{
Haploinsufficiency in the ANKSTB gene encoding AIDA-1 leads to a neurodevelopmental syndrome
}

\author{
Abigail U. Carbonell (10 ${ }^{1}$, Chang Hoon Cho ${ }^{1}$, Jaafar O. Tindi ${ }^{1}$, Pamela A. Counts ${ }^{2}$, Juliana C. Bates ${ }^{2}$, \\ Hediye Erdjument-Bromage ${ }^{3}$, Svetlana Cvejic ${ }^{1}$, Alana laboni ${ }^{4}$, Ifat Kvint ${ }^{5}$, Jenny Rosensaft ${ }^{6}$, Ehud Banne ${ }^{6}$, \\ Evdokia Anagnostou ${ }^{4}$, Thomas A. Neubert (10 ${ }^{3,7}$, Stephen W. Scherer ${ }^{8}$, Sophie Molholm ${ }^{1,2,9}$ \& \\ Bryen A. Jordan (1D) 1,9
}

Neurodevelopmental disorders, including autism spectrum disorder, have complex polygenic etiologies. Single-gene mutations in patients can help define genetic factors and molecular mechanisms underlying neurodevelopmental disorders. Here we describe individuals with monogenic heterozygous microdeletions in $A N K S 1 B$, a predicted risk gene for autism and neuropsychiatric diseases. Affected individuals present with a spectrum of neurodevelopmental phenotypes, including autism, attention-deficit hyperactivity disorder, and speech and motor deficits. Neurons generated from patient-derived induced pluripotent stem cells demonstrate loss of the ANKSTB-encoded protein AIDA-1, a brain-specific protein highly enriched at neuronal synapses. A transgenic mouse model of Anks $7 b$ haploinsufficiency recapitulates a range of patient phenotypes, including social deficits, hyperactivity, and sensorimotor dysfunction. Identification of the AIDA-1 interactome using quantitative proteomics reveals protein networks involved in synaptic function and the etiology of neurodevelopmental disorders. Our findings formalize a link between the synaptic protein AIDA-1 and a rare, previously undefined genetic disease we term ANKS1B haploinsufficiency syndrome.

\footnotetext{
${ }^{1}$ Dominick P. Purpura Department of Neuroscience, Albert Einstein College of Medicine, Bronx, 10461 NY, USA. ${ }^{2}$ Department of Pediatrics, Albert Einstein College of Medicine, Bronx, 10461 NY, USA. ${ }^{3}$ Department of Cell Biology and Kimmel Center for Biology and Medicine of the Skirball Institute, New York University School of Medicine, New York, 10016 NY, USA. ${ }^{4}$ Autism Research Centre, Bloorview Research Institute, Holland Bloorview Kids Rehabilitation Hospital, Toronto, M46 1R8 ON, Canada. ${ }^{5}$ Pediatric Neurology Clinic, Kaplan Medical Center, Hebrew University Hadassah Medical School, Rehovot 76100 , Israel. ${ }^{6}$ Genetics Institute, Kaplan Medical Center, Hebrew University Hadassah Medical School, Rehovot 76100, Israel. ${ }^{7}$ Department of Pharmacology, New York University School of Medicine, New York, 10016 NY, USA. ${ }^{8}$ Centre for Applied Genomics and McLaughlin Centre, Hospital for Sick Children and University of Toronto, Toronto, M56 OA4 ON, Canada. ${ }^{9}$ Department of Psychiatry and Behavioral Sciences, Albert Einstein College of Medicine, Bronx, 10461 NY, USA. Correspondence and requests for materials should be addressed to B.A.J. (email: bryen.jordan@einstein.yu.edu)
} 
$\mathrm{N}$ eurodevelopmental disorders, including autism spectrum disorder (ASD), attention-deficit/hyperactivity disorder (ADHD), and communication and motor disorders, have complex polygenic etiologies. These disorders are highly comorbid and share hereditary risk factors, suggesting that perturbations in pathways regulating brain development can result in a range of neurodevelopmental phenotypes ${ }^{1}$. In current models of autism heritability, common and rare variants interact with environmental factors to confer genetic risk ${ }^{2}$. Although de novo variants are often used to causally link individual genes to autism, inherited mutations in highly conserved genes also appear at higher rates in neurodevelopmental disorders and are noted to display variable penetrance ${ }^{3}$. Ultra-rare copy-number variations (CNVs) covering $>1$ kilobase are predicted to make a sizable contribution to risk for neurodevelopmental disorders 4 .

Recent analyses of genetic risk for autism identified $A N K S 1 B$ as a target gene due to rare genetic variants found in $\mathrm{ASD}^{5-7}$, predicted participation in gene networks dysregulated in $\mathrm{ASD}^{8,9}$, and salience in bioinformatic analyses of mouse phenomics ${ }^{10}$. Genomic and transcriptomic association studies of neuropsychiatric disorders implicate $A N K S 1 B$ in obsessivecompulsive disorder ${ }^{11,12}$, mood disorders ${ }^{13,14}$, and schizophrenia $^{15-18}$. ANKS1B is a large $\sim 1.3$ megabase gene located on human chromosome 12q23.1 that encodes AIDA-1 (APP intracellular domain associated 1), a protein initially suggested to regulate $\gamma$-secretase processing of amyloid precursor protein (APP $)^{19}$. We have shown that AIDA-1 is highly expressed in the brain, where it is enriched in hippocampal and cerebellar regions ${ }^{20}$ and is one of the most abundant proteins at neuronal synapses $^{21,22}$. AIDA-1 is specifically enriched at postsynaptic densities (PSDs), where it binds to N-methyl-D-aspartate receptors (NMDARs) and the adaptor protein PSD95 23 . Neuronal activity causes rapid translocation of AIDA-1 into the nucleus, resulting in changes in Cajal body stability and nucleolar morphology 23,24 . Recently, we found that postnatal deletion of AIDA1 from the forebrain led to reduced synaptic expression of the NMDAR subunit GluN2B and impaired NMDA-dependent longterm potentiation and long-term depression in the hippocampus $^{25}$.

Despite the literature suggesting an association between $A N K S 1 B$ and neurodevelopmental disease, no patients with confirmed loss of function in $A N K S 1 B$ have been previously identified. Here, we describe monogenic CNVs in $A N K S 1 B$ in individuals that display a spectrum of neurodevelopmental phenotypes, including ASD, ADHD, and speech and motor deficits. A newly generated mouse model of $A N K S 1 B$ haploinsufficiency syndrome exhibits behavioral correlates of the phenotypes observed in probands. Along with new evidence that AIDA-1 interacts with multiple regulators of neural development, our findings demonstrate that haploinsufficiency of $A N K S 1 B$ leads to a previously uncharacterized neurodevelopmental syndrome.

\section{Results}

ANKS1B deletion probands have neurodevelopmental disorders. We identified two families (EIN-1 and EIN-2) harboring monogenic microdeletions in $A N K S 1 B$ who had been referred for medical genetic evaluation due to various neurodevelopmental disorders, including autism, ADHD, speech apraxia, and motor delays (Table 1). We performed extensive neuropsychological testing and clinical interviews on all affected individuals from families EIN-1 and EIN-2 (Supplementary Data 1). Affected individuals displayed a pattern of developmental delays, oculomotor and oromotor irregularities, dysmetria, impaired finemotor dexterity, and problems with balance and gait. Subjects had a normal Full Scale Intelligence Quotient (FSIQ standard score
85-115), except for a female proband with speech apraxia (EIN$2-1$ ) whose composite FSIQ (standard score $=81$ ) was reduced by a verbal comprehension index (VCI) standard score of 73. Verbal impairments were more severe in female children (EIN-1-2 and EIN-2-1), consistent with their prior diagnosis of speech apraxia, and manifested in lower scores in VCI, expressive language, receptive language, and verbal memory compared to males. On a test of fine-motor dexterity and psychomotor speed, all children performed at least two standard deviations below the mean with their dominant hand $(z$-score -2.22 to -2.89$)$, whereas performance with the non-dominant hand was relatively spared. Deficits in oculomotor and fine-motor coordination contributed to poor or borderline performance in tasks requiring visual-motor integration. Consistent problems with speech and motor control contributed to variable performance on tests of attention and executive function (Supplementary Data 1).

In parent-rated assessments, both sets of parents uniformly endorsed low to average social skills in affected children (Supplementary Data 1). While neither of the affected female children met criteria for a social communication disorder, they showed deficits in affect recognition and theory of mind tasks. Affected children inherited the $A N K S 1 B$ microdeletion from a parent previously reported to have a mild (EIN-1-4) or normal (EIN 2-2) phenotype. However, we found that the affected parent in EIN-1, who had reported childhood motor delays, also displayed oculomotor, fine motor, visual motor, and gross motor deficits. The affected father in EIN-2, who had a previous diagnosis of ADHD, displayed impaired oculomotor control, visual-motor integration, and gross motor coordination (Supplementary Data 1). Moreover, a new diagnosis of autism was issued for one affected parent (EIN-1-4) at the conclusion of the study.

$A N K S 1 B$ copy-number variations have not previously been associated with a genetic syndrome. We therefore searched genotype-phenotype databases for individuals harboring similar CNVs. We identified ten additional probands in North America, Europe, and the Middle East harboring monogenic microdeletions in $A N K S 1 B$ through the Autism Speaks MSSNG project at the University of Toronto (TOR-1 and TOR-2), the DECIPHER project $^{26}$ (DEC-1,2,4,5,8,9,12), and the GeneMatcher online resource $^{27}$ (GEN-1) (Table 1 and Supplementary Data 2). We obtained clinical evaluations for five probands with monogenic deletions, as well as for four probands with CNVs in additional genes (Supplementary Data 2). Deletions in all individuals were identified during clinical testing through chromosomal microarray except for EIN-1-4 and EIN-2-2, which were confirmed by FISH, and TOR-1 and TOR-2, which were identified through whole-genome sequencing. All affected individuals were heterozygous for the ANKS1B microdeletion, and most deletions were inherited (Table 1 and Supplementary Data 2). We performed additional whole-exome sequencing in trios (proband and both parents) from the EIN-1 and EIN-2 families. No loss-of-function variants annotated with neurodevelopmental disorders in Clin$\operatorname{Var}^{28}$ were identified specifically in affected individuals (Supplementary Data 3). These results suggest that the monogenic deletions in $A N K S 1 B$ underlie the phenotypes identified in EIN-1 and EIN-2.

$A N K S 1 B$ deletion in these additional probands resulted in neurodevelopmental phenotypes consistent with our findings in the first two families (Table 1). ASD and ADHD were common diagnoses, along with speech and motor dyspraxia. Developmental delays, including delayed achievement of speech and motor milestones, were prevalent. Although intellectual disability was a variable finding, low IQ and global developmental delay were noted in a few patients. Several patients showed abnormal magnetic resonance imaging (MRI) findings, including T2 hyperintensities in various regions and dysgenesis or thinning 


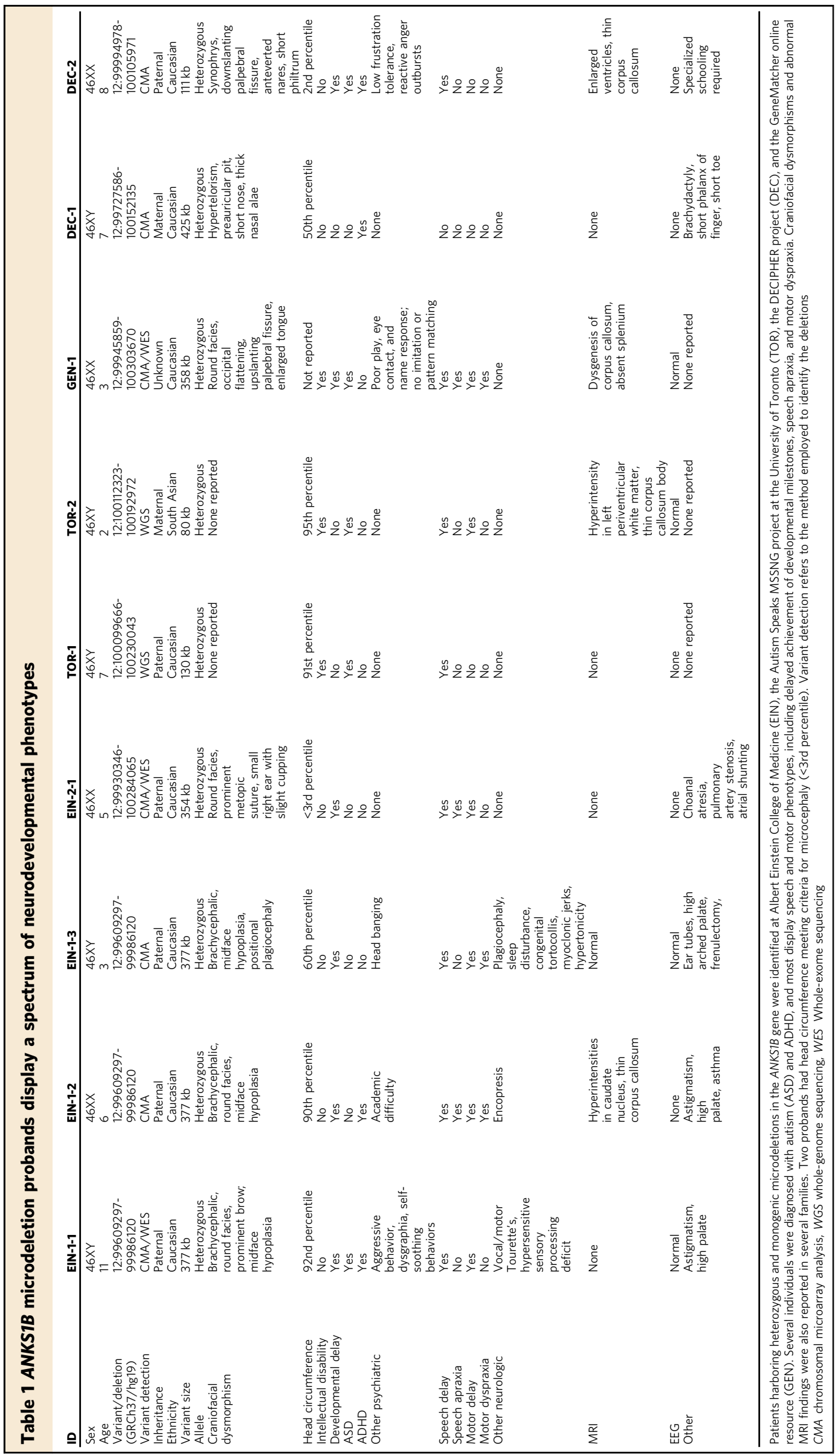


of the corpus callosum. Craniofacial dysmorphisms were also reported in several families, although specific features were not consistent among probands. Additional individuals harboring monogenic deletions in $A N K S 1 B$, as well as those with multiple CNVs affecting other genes (Supplementary Data 2), displayed similar neurodevelopmental phenotypes. All individuals identified in this study had received a clinical diagnosis before the age of 18 .

The rarity of patients with CNVs in this gene is likely due to intolerance of $A N K S 1 B$ loss of function in the population. Scarcity of $A N K S 1 B$ variants was a factor in its identification as a brain-enriched gene linked to autism ${ }^{6}$. Using a predictive model of haploinsufficiency, $A N K S 1 B$ is predicted to cause dominantlyinherited disease $\mathrm{e}^{29}$. From exome sequencing data obtained by the Exome Aggregation Consortium (ExAC), ANKS1B has a computed pLI (probability of loss-of-function intolerance) of 0.99 , indicating that fewer than $10 \%$ of the expected proteintruncating variants are observed for the gene $\mathrm{e}^{30}$. Furthermore, analysis of local missense constraint identifies two discrete regions in $A N K S 1 B$ with significantly less missense variation than expected: the seven $\mathrm{N}$-terminal exons (regional missense constraint $=0.451$, likelihood ratio test, $p=2.29 \mathrm{e}-7$ ), and the nine C-terminal exons (regional missense constraint $=0.412$, likelihood ratio test, $p=1.65 \mathrm{e}-8)^{31}$. These results indicate that variation in $A N K S 1 B$, including the $\mathrm{N}$-terminal region affected by most of the microdeletions we identified, is strongly selected against in the population.

$A N K S 1 B$ deletions lead to loss of AIDA-1 in patient neurons. $A N K S 1 B$ microdeletions in patients varied in size between 80-425 kilobases and targeted multiple exons in the $5^{\prime}$ region of the gene, predicting loss of AIDA-1 transcript and protein (Fig. 1a). However, $A N K S 1 B$ is a complex gene encoding more than 30 transcripts ${ }^{32}$, some of which reside outside the deletions. Assignation of exons is variable between genome repositories (NCBI, ENSEMBL), but all full-length $A N K S 1 B$ transcripts encode a protein containing six $\mathrm{N}$-terminal ankyrin repeats (ANK), two sterile alpha motif (SAM) domains, and a C-terminal phosphotyrosine binding (PTB) domain (AIDA-1B), while shorter isoforms lack the ankyrin repeats (AIDA-1D and AIDA-1C) (Fig. 1b). Western blots of postmortem human brain and mouse brain tissue show that in both species, AIDA-1B, AIDA-1D, and AIDA-1C are the main isoforms expressed in whole lysates and enriched in synaptic (SYN) and postsynaptic density (PSD) fractions. Knockdown of AIDA-1 expression in primary rat neurons using short-hairpin RNA (shRNA) ${ }^{25}$ confirms the specificity of AIDA-1 antibodies in human lysates since rodent and human AIDA-1 sequences are identical (Fig. 1c).

To determine how human $A N K S 1 B$ microdeletions affect AIDA-1 expression, we generated induced pluripotent stem cells (iPSCs) using peripheral mononucleated blood cells from probands and unaffected mothers in families EIN-1 and EIN-2 (Fig. 1d) through forced expression of transcription factors using Sendai virus ${ }^{33}$. Human-derived iPSCs were positive for pluripotency markers Oct-4 and Sox-2 by immunostaining (Fig. 1e). As AIDA-1 is enriched in neuronal synapses, we generated neurons by overexpressing the neuronal transcription factor neurogenin-2 $(\text { NGN2 })^{34}$. Differentiation of induced neurons (iNs) was confirmed by observed morphology and by immunostaining for the neuronal marker MAP2. Western blots of iNs showed that heterozygous $A N K S 1 B$ deletion in the EIN-2 proband reduced AIDA-1 expression compared to the unaffected mother (Fig. 1f, g). Similar results were obtained for the EIN-1 family (Supplementary Data 4). While we had expected a selective reduction in the full-length AIDA-1B isoform, our results show that deletions upstream of AIDA-1D and AIDA-1C transcripts similarly reduce expression of these shorter isoforms. Loss of major protein products suggests a model of genetic haploinsufficiency, but microdeletions could also result in abnormal RNAs and truncated polypeptides that contribute to disease. To address this possibility, we performed reverse transcription quantitative polymerase chain reaction (RT-qPCR) for exons along the $A N K S 1 B$ gene to identify abnormally generated transcripts. In both EIN-1 and EIN-2, families that contain distinct microdeletions, exons in $\mathrm{N}$-terminal, middle, and $\mathrm{C}$-terminal regions were downregulated in proband iNs (Fig. 1h). In addition, we performed western blots of iNs using AIDA-1 antibodies to other regions of the protein and observed similar changes (Supplementary Fig. 1). Altogether, these results strongly implicate genetic haploinsufficiency in disease etiology.

ANKS1B haploinsufficiency mouse model shows loss of AIDA1. Probands with $A N K S 1 B$ microdeletions exhibit neurodevelopmental disorders in childhood, suggesting dysregulation of early neural development. However, we had shown that shorter AIDA-1 isoforms are expressed postnatally in mice, reaching detectable levels 7-14 days after birth ${ }^{25}$. Western blots of mouse brain across development demonstrate that unlike AIDA-1D and AIDA-1C, the large AIDA-1B isoform is expressed from embryonic to adult stages (Fig. 2a and Supplementary Fig. 2a). Gene expression databases and postmortem human tissue analyses $^{35}$ show that $A N K S 1 B$ expression is highly brain-selective. In mouse tissue, we found that AIDA-1 isoforms are exclusively detected in brain, with the cerebellum expressing only the larger AIDA-1B isoform (Fig. 2b). Given the embryonic and neural expression pattern of AIDA-1, we used a transgenic Nestin-Cre mouse line to delete $A n k s 1 b$ from the developing central and peripheral nervous system starting at embryonic day $11^{36}$. Crossing Nestin-Cre mice to the Anks1b floxed mouse line previously developed ${ }^{25}$ revealed that homozygous AIDA-1 NestinCre knockout mice (Nestin-Cre;Anks1bflox/flox) do not survive beyond postnatal day 0 , highlighting a critical role for neural Anks $1 b$ in development. This observation of embryonic lethality is consistent with predicted loss-of-function intolerance in $A N K S 1 B^{30}$ and the lack of identified patients homozygous for $A N K S 1 B$ deletions.

Unlike homozygous Nestin-Cre knockouts, heterozygous Anks1b mice (Nestin-Cre;Anks1b ${ }^{\text {wt }} /$ flox, referred to here as Nestin-Het mice) were viable (Fig. 2c). Given that behavioral phenotypes have been reported in the Nestin-Cre transgenic line ${ }^{37}$, we used Nestin-Cre;Anks $1 b^{w t / w t}$ littermates (Nestin-WT mice) as controls for Nestin-Het mice in all experiments. Heterozygous Anks $1 b$ deletion resulted in a $\sim 50 \%$ reduction in AIDA-1 isoforms by western blot (Fig. 2c and Supplementary Fig. 2b, c) and reduced expression across $A n k s 1 b$ exons by RTqPCR similar to the changes observed in $A N K S 1 B$ microdeletion probands (Supplementary Fig. 2d). Gross characterization of Nestin-Het mice revealed small but significant sex-specific differences, with a decrease in weight, body length, and brain mass in male mice that was not significant in females (Fig. $2 \mathrm{~d}$ and Supplementary Data 4). Since AIDA-1 is enriched in the hippocampus and cerebellum, we performed a histological analysis of morphology in these brain regions. We found no significant changes in the size of these regions and no sexdependent differences by 2-way ANOVA (Supplementary Fig. 2e and Supplementary Data 4).

Anks1b mouse model recapitulates patient phenotypes. To test the effects of $A n k s 1 b$ deletion on an isogenic background, we performed a battery of behavioral assays on Nestin-Het mice 
a
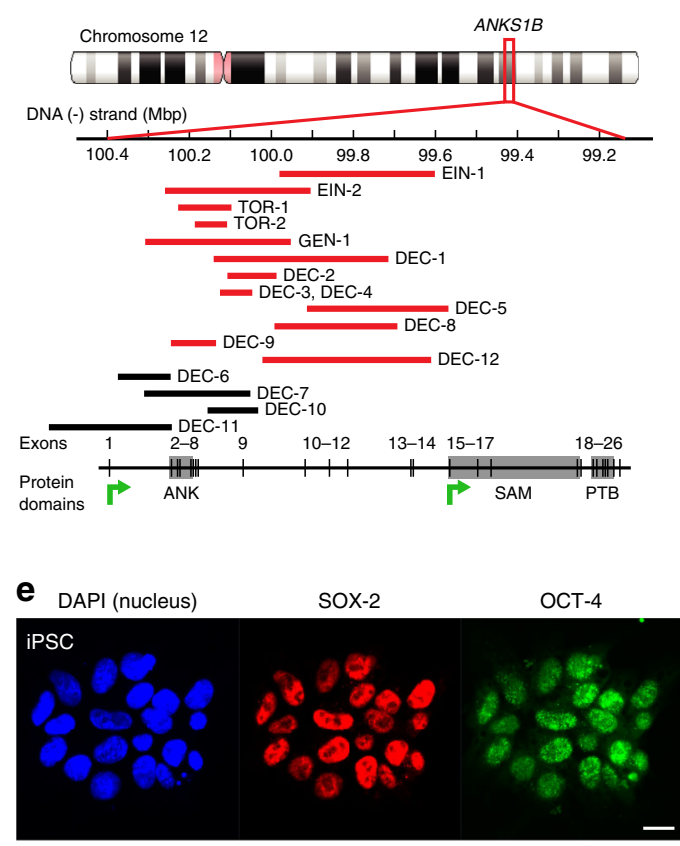

DAPI (nucleus)

MAP2

GFP

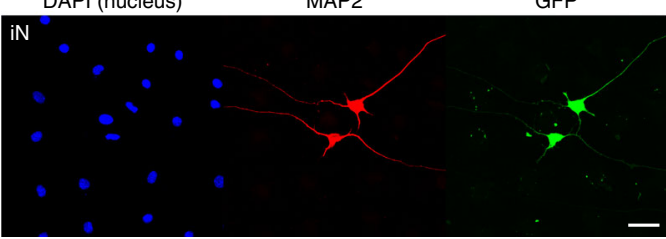

b

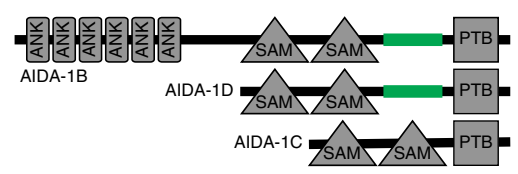

C

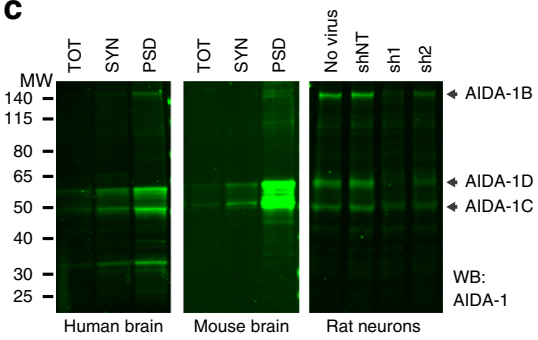

$\mathbf{f}$

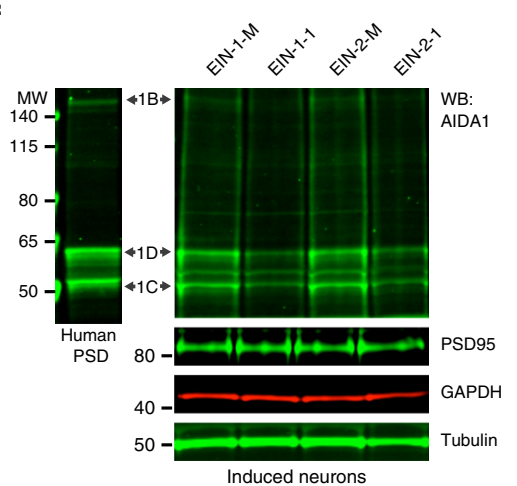

d
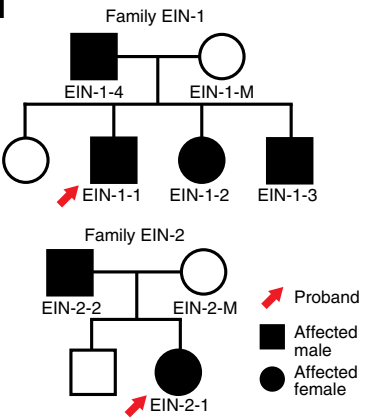

g

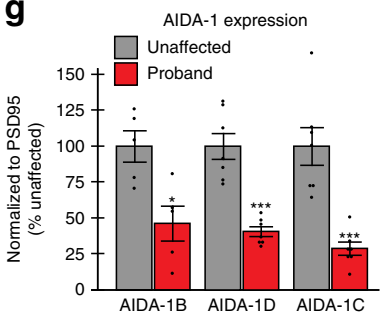

h

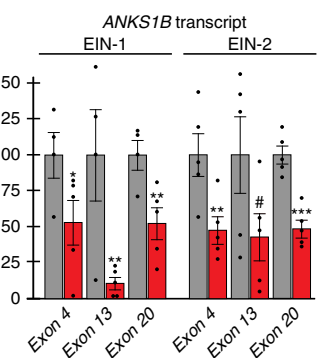

Fig. 1 ANKSIB microdeletions reduce AIDA-1 expression in patient-derived neurons. a Monogenic microdeletions (red bars) in patients span multiple exons in the ANKS1B gene. Black bars represent patients with additional CNVs in other genes. Green arrows represent putative transcription start sites. $\mathbf{b}$ ANKS1B encodes AIDA-1B, AIDA-1D, and AIDA-1C protein isoforms. c (Left) Western blots show that all three major AIDA-1 isoforms are expressed in total lysate (TOT) and enriched in synaptic (SYN) and postsynaptic density (PSD) fractions in postmortem human and mouse brain ( $5 \mu \mathrm{g}$ lysate). (Right) Lentivirusmediated knockdown of AIDA-1 in primary rat neurons (DIV 14-21) using two different shRNAs (sh1 and sh2 = AIDA-1 specific; shNT= scrambled control shRNA) confirms antibody specificity (20 $\mu$ g lysate). d Pedigree of EIN-1 and EIN-2 families. e (Top) IPSCs generated from probands (EIN-1-1 and EIN-2-1) and unaffected mothers (EIN-1-M and EIN-2-M) express Oct-4 and Sox-2 pluripotency markers. (Bottom) Induced neurons (iNs) generated from forced NGN2 expression (GFP) in iPSCs show typical neuronal morphology and express the mature neuronal marker MAP2. Nuclei from non-neuronal cells are co-cultured rat astrocytes added to improve neuronal viability and maturation. Scale bars $=10 \mu \mathrm{m}$. $\mathbf{f}$ Western blots of AIDA-1 and PSD95 show that AIDA-1 isoforms are significantly reduced in probands EIN-1-1 and EIN-2-1 compared to unaffected mothers EIN-1-M and EIN-2-M (10 $\mu$ g lysate). $\mathbf{g}$ Quantitation of AIDA-1 expression normalized to neuronal marker PSD95 from Family EIN-2. $N=5-7$ biological replicates. Bar graphs show mean \pm SEM, two-sided Student's $t$-test, ${ }^{*} p<0.05^{* * *} p<0.001$. h RT-qPCR of exons 4,13 , and 20 spanning the ANKS1B gene and normalized to neuronal marker MAP2 are reduced in probands from both families. $N=4-5$ biological replicates for each family. Bar graphs show mean $\pm \mathrm{SEM}$, one-sided Student's $t$-test, ${ }^{\star} p<0.05,{ }^{\star \star} p<0.01$, ${ }^{\star \star \star} p<0.001,{ }^{\#} p=0.052$

across domains relevant to clinical features of ANKS1B haploinsufficiency syndrome (Fig. 3). Using the Behavioral Spectrometer, a system validated to assess aberrant activity patterns in mouse models of $\mathrm{ASD}^{38}$, we found that Nestin-Het mice covered greater distances and had increased episodes of running activity compared to Nestin-WT controls, consistent with a hyperactivity phenotype (Fig. 3a). No significant differences were observed in the number of grooming episodes, a correlate of stereotypies in ASD, or in orienting and rearing (Supplementary Fig. 3a). However, Nestin-Het mice showed an increase in number of visits, time, and percentage of total track in the center square of an open field, despite no difference in rearing (risk assessment), suggesting a reduction in anxiety. To test anxiety-related behaviors more robustly, we used the elevated plus maze, an assessment of unconditioned approach-avoidance conflict ${ }^{39}$. NestinHet mice spent increased time and distance in the open arms of the maze as a percentage of total track, confirming a decreased anxiety phenotype (Fig. 3b and Supplementary Fig. 3b). This effect was not generalizable to another measure of mood dysregulation, the Porsolt forced swim test of learned helplessness in depression (Supplementary Fig. 3c).

In the three-chamber test of social approach, an assay widely used to evaluate mouse models of autism ${ }^{40}$, Nestin-Het mice showed significantly reduced preference for a conspecific mouse over an inanimate object, and notably demonstrated borderline preference $(51.1 \pm 1.7 \%$, mean \pm SEM) for social interactions (Fig. 3c). Nestin-Het mice had a significantly higher likelihood of failing the social preference test as defined by spending less than $50 \%$ of total exploration time with the animal (Supplementary Fig. 3d). While Nestin-WT mice spent significantly more time exploring the animal than the object, Nestin-Het mice showed no difference between time spent sniffing the animal and time spent with the object (Supplementary Fig. 3d). While impaired sociability is the principal behavioral deficit observed in 
a

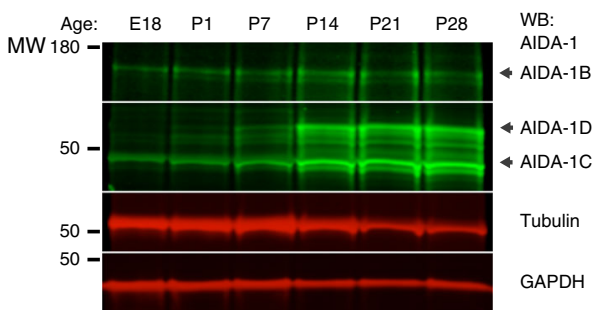

b

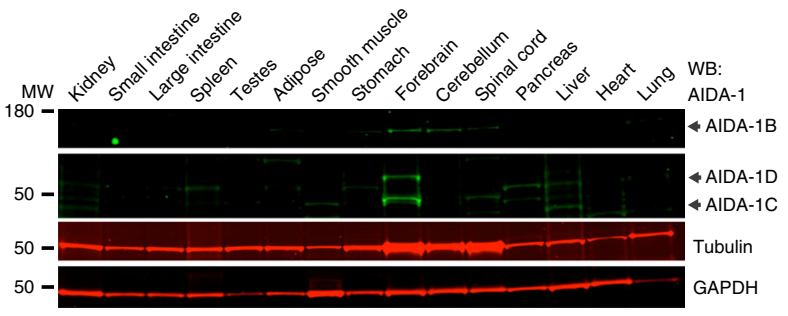

C
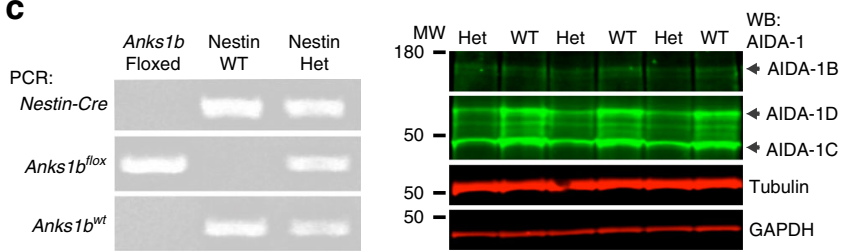

d
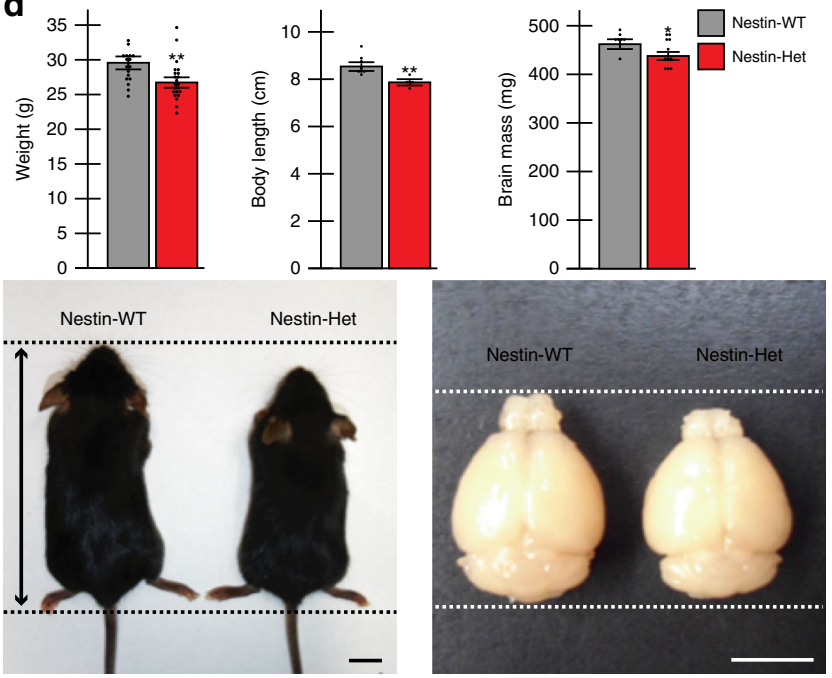

Fig. 2 Heterozygous Anks $1 b$ knockout mice are viable and show reduced AIDA-1 expression. a Western blots show AIDA-1B is expressed in mouse brain tissue from embryonic development to adulthood. AIDA-1D and $1 C$ expression increases until reaching stable levels in the adult brain. Tubulin and GAPDH levels are shown as loading controls (20 $\mu \mathrm{g}$ lysate). $\mathrm{E}=$ embryonic day, $\mathrm{P}=$ postnatal day. $\mathbf{b}$ AIDA-1 isoforms are selectively expressed in whole mouse brain and cerebellum ( $20 \mu \mathrm{g}$ lysate). c (Left) PCR genotyping to identify Nestin-Het mice. (Right) Western blots show reduced expression of AIDA-1 isoforms in Nestin-Het mice (20 $\mu$ g lysate). d Male Nestin-Het mice show decreased total weight (26.7 $\pm 0.6 \mathrm{~g}$, mean \pm SEM) compared to Nestin-WT controls $(29.5 \pm 0.8 \mathrm{~g}) ; N=41$ mice. Body length was also reduced $(7.88 \pm 0.09 \mathrm{~cm})$ compared to controls $(8.55 \pm$ $0.14 \mathrm{~cm}$ ); $N=13$ mice, scale bar in representative image $=1 \mathrm{~cm}$. Brain mass was also reduced (Nestin-Het $=442.7 \pm 8.5 \mathrm{mg}$, Nestin-WT $=467.5 \pm 6.2$ $\mathrm{mg}$; $N=19$ mice, scale bar in representative image $=0.5 \mathrm{~cm}$. Bar graphs show mean \pm SEM, two-sided Student's $t$-test, ${ }^{\star} p<0.05$, ${ }^{\star *} p<0.01$. No statistically significant differences were observed in female mice (Supplementary Data 4) autism mouse models ${ }^{40}$, we performed additional testing for sensorimotor deficits, which often accompany an ASD diagnosis and are notable features of $A N K S 1 B$ haploinsufficiency syndrome. In measures of the acoustic startle reflex, Nestin-Het mice showed a robust increase in the peak magnitude of the startle response (Fig. 3d). They also demonstrated marked impairments in prepulse inhibition (PPI) of the response, a measure of sensorimotor gating (Fig. 3d and Supplementary Fig. 3e). Deficits in startle and PPI have been described in several mouse models of autism ${ }^{40}$. Nestin-Het mice displayed significant deficits in finemotor dexterity assayed by the adhesive removal test ${ }^{41}$ (Fig. 3e). Gross motor coordination was comparable to controls as measured by slips on a wooden balance beam, although we observed a trend toward increased slips in the Nestin-Het mice.

Despite our previous finding that AIDA-1 regulates synaptic plasticity in the hippocampus ${ }^{25}$, Nestin-Het mice showed no cognitive deficits. In the object placement test, a hippocampusdependent learning task ${ }^{39}$, Nestin-Het mice showed no difference in preference for a novel object location compared to controls, a result consistent between retention intervals in which controls passed (short delay) or failed (long delay) (Fig. 3f). Statistical analyses for the 46-97 adult mice tested demonstrate robust results with high power for tests in which differences were significant. No sex differences were observed in any of the behaviors evaluated (Fig. 3g and Supplementary Data 5). To determine whether similar phenotypes could be observed earlier in development ${ }^{42}$, we tested Nestin-Het pups at pre-weaning ages. To test for the emergence of social, sensory, and motor deficits, we performed homing, negative geotaxis, and acoustic startle tests in pups from P10 to P16. No deficits or delays were observed for Nestin-Het mice in these developmental milestones compared to Nestin-WT and other littermate control groups (Supplementary Fig. 3f and Supplementary Data 5). To test for social communication deficits, we analyzed ultrasonic vocalizations (USVs) recorded from maternally separated pups at P8 and P10. No significant changes in frequency, syllable counts and intervals, or syllable repertoires were detected between NestinHet pups and three other littermate control groups (Supplementary Fig. 3g-i).

AIDA-1 interactome reveals novel cellular roles for ANKS1B. Our findings demonstrate that loss of AIDA-1 expression leads to neurobehavioral phenotypes in both human and mouse. To obtain insight into the molecular mechanisms that link AIDA-1 to disease, we identified the AIDA-1 interactome in mouse brain using quantitative proteomic methods (Fig. 4a). We performed ten unique immunoprecipitations (IPs) from mouse brain lysates using control IgGs, AIDA-1 IgGs, and antibodies to unrelated synaptic proteins crosslinked to protein G-agarose or magnetic beads. Each eluted IP sample was labeled with a unique isobaric tag within a 10-plex set of tandem mass tags ${ }^{43}$. This approach allowed us to accurately quantify identified peptides, simultaneously analyze all samples to reduce variability associated with mass spectrometry (MS) analysis, and rigorously control for nonspecific binding by normalizing to control IgG samples and comparing to unrelated protein interactomes (Supplementary Data 6a). We used a commercially available antibody (C-10, Santa Cruz Biotechnologies), as well as in-house antibodies known to immunoprecipitate AIDA-1 (1A11 and 2B22) (Fig. 4b). We defined the AIDA-1 interactome as proteins coimmunoprecipitated by AIDA-1 antibodies that were identified and quantified by $\geq 3$ peptides and enriched $>1.4$-fold over background in agarose experiments, or $>3.0$-fold in magnetic bead experiments (Supplementary Data 6b). Determination of these cutoff values is explained in Methods. To gauge the 
specificity of the AIDA-1 interactome, we analyzed the overlap between AIDA-1 and unrelated interactomes using two-sided Fisher's exact test. Since co-IP for synaptic proteins X and Y are likely to yield similar protein populations, we used a stringent statistical background for the hypergeometric distribution limited to proteins enriched in any co-IP sample (255 total proteins). As expected, the interactors obtained using a mixture of AIDA-1 antibodies (AIDA-1 Mix Agarose) showed significant overlap only with those from other AIDA-1 samples (Fig. 4c).

We analyzed the AIDA-1 interactome using StringDB ${ }^{44}$ for a general overview of gene ontology (GO) enrichments (Fig. $4 \mathrm{~d}$ and Supplementary Data 7). The interactome contained proteins from cellular components where AIDA-1 is known to localize, including the synaptic and postsynaptic compartments of neuronal projections. However, the most significant enrichment was in components of membrane-bound vesicles (Fig. 4e). In line with this localization, our previous work demonstrated impaired NMDAR subunit trafficking through the endoplasmic reticulum (ER) in Anks1b conditional knockout mice ${ }^{25}$. Although the mechanism of this regulation is unknown, we had previously shown that AIDA-1 is associated with intracellular vesicles in the presynaptic and postsynaptic compartments ${ }^{20}$. To confirm the vesicular distribution of $A N K S 1 B$-encoded proteins, we performed full subcellular fractionation of postmortem human brain and mouse brain tissue. Western blots show that AIDA-1 isoforms are enriched in microsomes derived from light membrane fractions, as well as PSD fractions (Supplementary Fig. 4). Characterization of these microsomal fractions shows that they are enriched in the endoplasmic reticulum marker calnexin ${ }^{45}$ as well as small GTPases (Rab11) that regulate trafficking through recycling endosomes ${ }^{46}$. To validate novel AIDA-1 interactors, we performed independent co-IP experiments in mouse brain lysates and confirmed the association of AIDA-1 with Git $1^{47}$, Itsn 1 and Ap2a1/2 $2^{48}$, Asap $1^{49}$, and Srgap2 ${ }^{50}$, proteins that regulate membrane trafficking and synaptic development (Fig. 4f).

AIDA-1 interactome links ANKS1B to development and disease. We identified the most significantly regulated diseases and functions in the AIDA-1 interactome (Supplementary Data 8a), then performed higher-order analyses to categorize these annotations using Ingenuity Pathway Analysis (IPA, QIAGEN Bioinformatics) (Fig. 5a). Consistent with clinical findings in ANKS1B haploinsufficiency syndrome, Neurological Disease and Developmental Disorder were among the top hits in the category of Diseases and Disorders (Supplementary Data 8b). In the category of Physiological Systems, the most significant effects were found on development, including nervous system development and function. Top molecular functions of the interactome converged on cellular processes, including cell morphology and cellular development. Advanced pathway construction in IPA identified a functional network with predicted functions in cell-to-cell signaling and interaction, cell morphology, and nervous system development and function as the top-scoring network (Fig. 5b and Supplementary Data 8c). Inspection of individual proteins in this network revealed known AIDA-1 interactors, including $\mathrm{PSD}^{23}{ }^{23}$, SynGAP1 ${ }^{51}$, and SNX2752. These and other molecules converge on the NMDA receptor as a major node in the pathway. Based on this network analysis, the interaction of AIDA-1 with proteins that regulate vesicle transport (Fig. 4e, f and Supplementary Data 4), and our work linking AIDA-1 to NMDAR function $^{25}$, we measured surface expression of the NMDAR subunits GluN2B and GluN2A in iPSC-derived neurons using immunofluorescence. Contrary to our findings in forebrain $A n k s 1 b$ knockout mice ${ }^{25}$, we found no reduction in surface GluN2B levels in proband neurons. However, we found that
GluN2A subunits showed a significant increase in surface expression, which is consistent with our previous findings (Fig. 5c). No changes in overall GluN2B and GluN2A levels were detected by western blot, suggesting that $A N K S 1 B$ haploinsufficiency leads to aberrant trafficking of NMDAR subunits in patient-derived neurons. These results indicate that altered NMDAR localization is a possible mechanism of disease in $A N K S 1 B$ haploinsufficiency syndrome. From the identity of individual molecules to their predicted roles in disease, our analysis of the AIDA-1 interactome predicts participation of $A N K S 1 B$ in cellular processes crucial to normal development and implicated in the etiology of neurodevelopmental disorders.

\section{Discussion}

In this study, we discovered that microdeletions in the ANKS1B gene encoding AIDA-1 lead to a neurodevelopmental syndrome characterized by autism, ADHD, and speech and motor impairment. Ours is the first to report patients with monogenic deletions in $A N K S 1 B$ and establish the contributions of these rare CNVs to disease. None of the variants we report have been identified in control populations ${ }^{30}$. However, most $A N K S 1 B$ deletions were inherited from parents reported to have normal or mild phenotypes (Table 1 and Supplementary Data 2). Variable penetrance is a common feature of CNVs in neurodevelopmental disorders and could be explained by inherited risk factors unmasked by deletions, or by compensation strategies employed throughout development ${ }^{3,4}$. Through comprehensive clinical interviews and testing, we found previously undiagnosed neurodevelopmental phenotypes in affected fathers from families EIN-1 and EIN-2. In both cases, fathers showed impairments in similar domains as their affected children and other probands, demonstrating that the phenotype segregated with the $A N K S 1 B$ deletion. The degree to which the deletion impaired adaptive function was related to developmental age: parents rated younger affected children as having more difficulty with daily function compared to older children. Our results highlight the need for deep clinical phenotyping in addition to genomic sequencing to identify links between CNVs and phenotypes, especially in spectrum disorders with variable presentations and developmental compensation. Despite the range of phenotypes and the rarity of $A N K S 1 B$ mutations, we identified 12 families with shared features, supporting a causal link between $A N K S 1 B$ deletion and the clinical syndrome. We also identified $A N K S 1 B$ deletions in patients with CNVs overlapping other genes. Similarities between the neurodevelopmental disorders in these individuals and those with monogenic $A N K S 1 B$ deletions suggests that $A N K S 1 B$ haploinsufficiency contributes to clinical presentation in these patients.

$A N K S 1 B$ encodes the protein AIDA-1, which is highly enriched in the brain and specifically expressed in neurons. We induced differentiation of iPSCs into excitatory neurons to enable further investigation of synaptic dysfunction and aberrant neuronal development, which are proposed mechanisms for neurodevelopmental disorders ${ }^{53}$. Using neurons from different families, we correlated microdeletions overlapping distinct ANKS1B exons with similar reductions in encoded AIDA-1 protein (Fig. If and Supplementary Data 4). Expression of all major AIDA-1 isoforms and $A N K S 1 B$ transcripts are reduced, including those with putative transcription start sites downstream of microdeletions. Reduced AIDA-1D and AIDA-1C protein isoforms may reflect the loss of unidentified cis-acting regulatory elements in the deleted $\mathrm{N}$-terminal regions of the ANKS1B gene. Alternatively, AIDA-1 isoforms may stabilize each other, such that loss of the larger protein leads to the degradation of smaller isoforms. Additional work will be needed to elucidate this potential isoform-dependent regulation of $A N K S 1 B$ expression. 


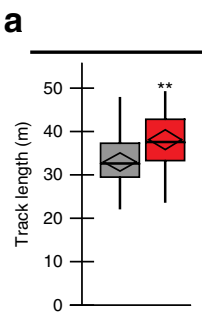

b

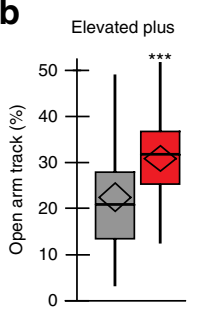

e

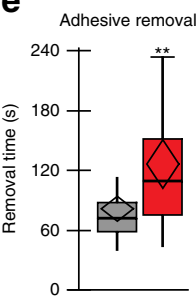

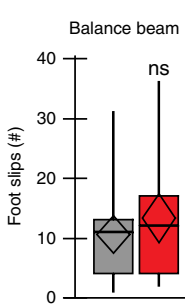

Behavioral spectrometer

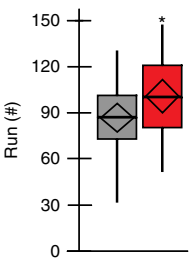

C Three chamber

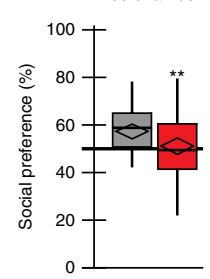

Acoustic startle
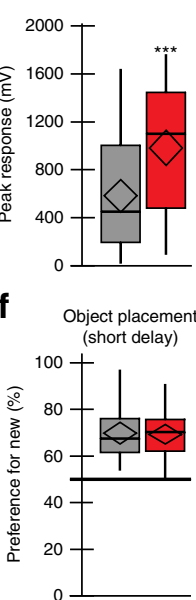

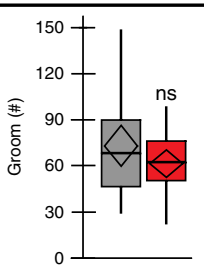

$\square^{\text {Nessin-WT }}$

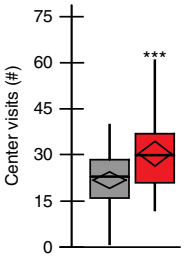

Prepulse inhibition $100 \div$

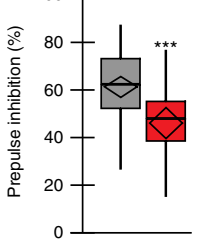

Object placement

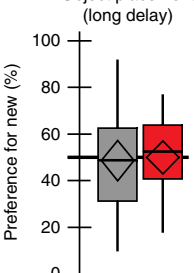

g

\begin{tabular}{|c|c|c|c|c|c|c|c|c|c|}
\hline & Group & $N$ & Mean & SEM & & Group & $N$ & Mean & SEM \\
\hline \multirow{2}{*}{$\begin{array}{l}\text { Total track } \\
\text { length }(\mathrm{cm})\end{array}$} & WT & $21 \mathrm{M} 20 \mathrm{~F}$ & 3301.3 & 103.3 & \multirow{2}{*}{$\begin{array}{c}\text { Acoustic } \\
\text { startle (mV) }\end{array}$} & WT & $27 \mathrm{M} 22 \mathrm{~F}$ & 581.35 & 67.45 \\
\hline & Het & $19 \mathrm{M} 18 \mathrm{~F}$ & 3813.1 & 114.2 & & Het & $26 \mathrm{M} 22 \mathrm{~F}$ & 981.10 & 72.53 \\
\hline \multirow{2}{*}{$\begin{array}{c}\text { Running } \\
\text { (episodes) }\end{array}$} & WT & $13 \mathrm{M} 10 \mathrm{~F}$ & 87.78 & 4.65 & \multirow{2}{*}{$\begin{array}{c}\text { Prepulse } \\
\text { inhibition (\%) }\end{array}$} & WT & $27 \mathrm{M} 22 \mathrm{~F}$ & 61.27 & 2.15 \\
\hline & Het & $15 \mathrm{M} 8 \mathrm{~F}$ & 103.18 & 4.62 & & Het & $26 \mathrm{M} 22 \mathrm{~F}$ & 46.00 & 3.25 \\
\hline \multirow{2}{*}{$\begin{array}{l}\text { Grooming } \\
\text { (episodes) }\end{array}$} & WT & $13 \mathrm{M} 10 \mathrm{~F}$ & 74.13 & 6.51 & \multirow{2}{*}{$\begin{array}{l}\text { Adhesive } \\
\text { removal (s) }\end{array}$} & WT & $19 \mathrm{M} 12 \mathrm{~F}$ & 81.10 & 6.09 \\
\hline & Het & $15 \mathrm{M} 8 \mathrm{~F}$ & 60.35 & 4.18 & & Het & $16 \mathrm{M} 12 \mathrm{~F}$ & 125.81 & 11.98 \\
\hline \multirow{2}{*}{$\begin{array}{c}\text { Center square } \\
\text { (visits) }\end{array}$} & WT & $21 \mathrm{M} 20 \mathrm{~F}$ & 21.85 & 1.33 & \multirow{2}{*}{$\begin{array}{l}\text { Balance beam } \\
\text { (slips) }\end{array}$} & WT & $13 \mathrm{M} 10 \mathrm{~F}$ & 10.61 & 1.50 \\
\hline & Het & $19 \mathrm{M} 18 \mathrm{~F}$ & 30.41 & 1.99 & & Het & $15 \mathrm{M} 8 \mathrm{~F}$ & 13.30 & 1.96 \\
\hline \multirow{2}{*}{$\begin{array}{c}\text { Open arm track } \\
(\%)\end{array}$} & WT & $27 \mathrm{M} 22 \mathrm{~F}$ & 22.48 & 1.52 & \multirow{2}{*}{$\begin{array}{l}\text { Preference-40 } \\
\min (\%)\end{array}$} & WT & $13 \mathrm{M} 10 \mathrm{~F}$ & 69.82 & 2.22 \\
\hline & Het & $26 \mathrm{M} 22 \mathrm{~F}$ & 30.88 & 1.36 & & Het & $15 \mathrm{M} 8 \mathrm{~F}$ & 69.36 & 2.06 \\
\hline \multirow{2}{*}{$\begin{array}{c}\text { Social } \\
\text { preference (\%) }\end{array}$} & WT & $27 \mathrm{M} 22 \mathrm{~F}$ & 57.31 & 1.53 & \multirow{2}{*}{$\begin{array}{l}\text { Preference-90 } \\
\min (\%)\end{array}$} & WT & $13 \mathrm{M} 10 \mathrm{~F}$ & 48.52 & 4.21 \\
\hline & Het & $26 \mathrm{M} 22 \mathrm{~F}$ & 51.14 & 1.72 & & Het & $15 \mathrm{M} 8 \mathrm{~F}$ & 49.84 & 3.40 \\
\hline
\end{tabular}

Patient-derived iNs and Nestin-Het mice both demonstrate reduction in all major isoforms and transcripts (Fig. $1 \mathrm{~g}, \mathrm{~h}$ and Supplementary Fig. $2 c$, d). This suggests broad dysfunction in AIDA-1-regulated processes across brain regions: ANKS1B transcripts are differentially spliced in Wernicke's area, important for language development, and the prefrontal cortex, implicated in neurodevelopmental disorders including autism ${ }^{54}$. AIDA-1 is strongly expressed in the cerebellum ${ }^{20}$, especially the large isoform AIDA-1B (Fig. 2b). Anks1b transcripts are present in Purkinje cells of the developing cerebellum ${ }^{55}$, consistent with the striking motor phenotypes in patients and the emerging role of the cerebellum in language and social development ${ }^{56}$. Although gross characterization of cerebellar size revealed no significant changes in Anks1b Nestin-Het mice (Supplementary Fig. 2e), our gene expression and behavioral data demonstrate that ANKS1B deletions could affect development and function in brain regions crucial for motor coordination and social communication.

Heterozygous Ankslb deletion in mice produced impaired social interactions, altered activity patterns, and sensorimotor deficits reminiscent of $A N K S 1 B$ haploinsufficiency syndrome. We did not observe the impairment in hippocampus-dependent spatial memory that would be expected after loss of a gene
Fig. 3 Anks $1 b$ heterozygous mice recapitulate phenotypes in ANKS1B haploinsufficiency syndrome. a In the Behavioral Spectrometer, Nestin-Het mice covered more track in 9 min and had more episodes of running than Nestin-WT controls. Reduction in grooming behavior was not statistically significant. Nestin-Het mice visit the center square of an open field more often than controls. b Nestin-Het mice showed a robust reduction in avoidance behaviors, covering more track in the open arms as a percentage of total track in open and closed arms of an elevated plus maze. $\mathbf{c}$ In the three-chamber test, Nestin-Het mice show significantly reduced and borderline preference for a conspecific mouse over an inanimate object. d Peak magnitude of the acoustic startle reflex is robustly increased in Nestin-Het mice. Sensorimotor gating measured by the percentage of prepulse inhibition (prepulse stimulus 40 ms before startle stimulus) is significantly reduced. e In a test of fine-motor dexterity, Nestin-Het mice require more time to remove adhesive from the forepaw. Nestin-Het mice do not exhibit significant deficits in gross motor coordination as measured by slips on a balance beam. $\mathbf{f}$ Nestin-Het mice do not show learning deficits in the object placement test, a hippocampus-dependent memory assay. Both groups averaged above a passing score ( $>50 \%$ preference for new location) using a 40-min retention interval. Raising the task difficulty by increasing the retention interval to $90 \mathrm{~min}$ did not result in a difference: mean values indicate that mice of both genotypes failed the test at $90 \mathrm{~min}$ ( $<50 \%$ preference). Reference line indicates $50 \%$ preference. Box plots show the mean and $95 \%$ confidence intervals (black diamond), median (black line), 25th-75th quantile (gray or red bar), and range (black whiskers). If two-way ANOVA showed significant main effect of genotype, post hoc two-sided Student's $t$-test was performed, ${ }^{\star} p<0.05,{ }^{\star \star} p<0.01$, ${ }^{\star \star \star} p<0.001, \mathrm{~ns}=p>0.05$. $\mathbf{g}$ Numbers and sex of mice tested with the measures for each behavioral assay are shown as the mean and SEM. No sex-dependent effects of genotype were observed in any behavioral test by two-way ANOVA (Supplementary Data 5)

required for hippocampal synaptic plasticity ${ }^{25}$. Heterozygous knockdown of Anks $1 b$ may not have been sufficient to produce changes in plasticity, since our previous work used a homozygous forebrain knockout model. Moreover, intellectual disability is an inconsistent feature in patients. Motor deficits in speech production and fine-motor control are prominent features of the syndrome, reflected in reduced dexterity in Nestin-Het mice. Gross motor coordination was not significantly impaired in the balance beam test, although a trend was observed toward increased slips. Impaired sociability, altered approach-avoidance behaviors, hyperactivity, increased startle sensitivity, and reduced prepulse inhibition have all been described in mouse models of autism, including Fmr1 knockout and the BTBR strain ${ }^{40}$. Our findings of neurobehavioral abnormalities are largely consistent with a previous evaluation of ketamine sensitivity in a homozygous Anks $1 b$ deletion mode ${ }^{57}$. Notably, we found that heterozygous deletion with $\sim 50 \%$ residual AIDA- 1 expression is sufficient to observe these behavioral effects, supporting an $A N K S 1 B$ haploinsufficiency model in the etiology of the clinical syndrome. In contrast to ANKS1B deletion probands, Anks $1 b$ Nestin-Het mice did not demonstrate social, sensory, or motor impairments in early development (Supplementary Fig. $3 \mathrm{f}-\mathrm{i}$ ). However, developmental stages in mice correlate imperfectly with the timing of human development, and an initial period of normal development has been described in other mouse models of syndromic neurodevelopmental disorders ${ }^{58}$. Since the Nestin-Het model essentially reproduces a heterozygous Anks $1 b$ microdeletion on an isogenic background, our results strengthen the causal link between ANKS1B deletion and neurodevelopmental disorders in probands.

Through co-immunoprecipitation and quantitative mass spectrometry, we defined the AIDA-1 interactome with 
significant reproducibility. The novel methodology we present here, involving simultaneous analysis of multiple internal controls, increases our confidence in the AIDA-1 interactors identified. An unbiased approach using whole brain homogenate enabled us to identify novel interactors and striking enrichment in components of membrane-bound vesicles, including Rab family regulators of recycling and contents of the AP-2 endocytic complex. Fractionation and co-immunoprecipitation with key regulators of vesicle transport validated this approach. Higherorder analysis of the AIDA-1 interactome in IPA traced converging functions of $A N K S 1 B$ in normal development and disease-related processes (Supplementary Data $8 \mathrm{~b}$ ). Comparing our proteomic data to transcriptomic and genomic studies identifying $A N K S 1 B$ as a gene of interest leads to several intriguing parallels. In diseases and disorders, neurological disease is the top category: aside from the neurodevelopmental and neuropsychiatric disorders in ANKS1B haploinsufficiency syndrome, literature also suggests a role in dementia ${ }^{59}$ and epilepsy ${ }^{60}$. In physiological systems, major roles for the AIDA-1 interactome in organismal and embryonic development is consistent with $A N K S 1 B$ influence on body size ${ }^{61}$, gestational development ${ }^{62}$, and our current findings of reduced body mass and perinatal lethality in Anks $1 b$ knockout mice. For nervous system development and function, the annotation of highest significance is neuritogenesis (IPA, one-sided Fisher's exact test, $p=1.56 \mathrm{e}-15$ ), which is represented in all top molecular functions of the AIDA-1 interactome. Finally, top canonical pathways regulated by the AIDA-1 interactome include axon guidance signaling and endocytic pathways, which are supported by our co-IP and fractionation results. These results place $A N K S 1 B$ among genes that link endosomal trafficking to neurodevelopmental disorders ${ }^{63,64}$.

Analysis of the top network in the AIDA-1 interactome reconciles known and novel roles for AIDA-1: postsynaptic scaffolds (PSD95, DLGAP3), vesicle components (Snx27, AP-2), enzymatic switches (SynGAP1, AAK1), and membrane proteins (NMDAR, Contactin-1) are all major players. The pathway features prominent regulators of synaptic function implicated in neurodevelopmental disorders, including NMDARs (autism) ${ }^{65}$, SynGAP1 (autism and ID) ${ }^{66}$, and Snx27 (Down Syndrome) ${ }^{67}$. Given that AIDA-1 participates in the localization of GluN2A and GluN2B ${ }^{25}$, we tested the hypothesis that changes in NMDAR expression underlie $A N K S 1 B$ haploinsufficiency syndrome. Although surface GluN2A was significantly increased in probandderived neurons, we did not observe changes in GluN2B. This discrepancy may reflect dose-dependent or synapse-specific effects: in contrast to homozygous AIDA-1 forebrain knockout mice, all human microdeletions are heterozygous, proband neurons retain $\sim 50 \%$ of AIDA-1, and total surface expression was measured (Fig. 1g). Moreover, GluN2B is the primary subunit expressed in iPSC-derived neurons ${ }^{68}$ : regulatory mechanisms maintaining GluN2B expression could compensate for partial loss of AIDA-1. Divergent mechanisms may regulate NMDAR subunit composition in humans and mice, highlighting the importance of developing this human-based model ${ }^{69}$ of $A N K S 1 B$ haploinsufficiency syndrome. Overall, we showed that ANKS1B haploinsufficiency leads to changes in NMDAR subunit localization, validating the functions predicted by our previous work and our current analysis of the AIDA-1 interactome.

We identified $A N K S 1 B$ as a crucial gene for human development in which rare loss-of-function variants cause neurodevelopmental disorders, including autism, ADHD, and deficits in speech and motor function. Through proteomic analysis, we defined a network of molecular pathways through which AIDA-1 interactors regulate neuronal function and identified a specific deficit in NMDAR subunit localization as a possible mechanism of disease. By generating neurons from patients with ANKS1B haploinsufficiency syndrome and the Anks1b Nestin-Het mouse model, we have developed tools to further probe the mechanisms by which AIDA-1 enables normal brain development and $A N K S 1 B$ haploinsufficiency contributes to neurodevelopmental disorders.

\section{Methods}

Human subjects and clinical phenotyping. Clinical phenotyping received ethical approval by the Institutional Review Board (IRB) at Albert Einstein College of Medicine in IRB protocol \#2011-320 to S.M. Informed consent was obtained from all participants. Patients were identified through direct contact (EIN-1 and EIN-2), the Autism Speaks MSSNG project (TOR-1 and TOR-2), the DECIPHER database (DEC-1 through DEC-12), and clinicians contacted by the GeneMatcher online resource (GEN-1). Families EIN-1 and EIN-2 were enrolled in a protocol approved by the Institutional Review Board at Albert Einstein College of Medicine. Clinical interviews and exams, neuropsychological tests, and family questionnaires were administered at the Albert Einstein College of Medicine Human Clinical Phenotyping Core by licensed neuropsychologists. Evaluation of children was conducted in the morning, while evaluation of adults was conducted from morning until afternoon. All subjects were given a lunch break at noon, and additional breaks were provided when requested. Subjects completed a neuropsychological battery consisting of tests appropriate to developmental age. Tests generated a standard score $($ mean $=100$, standard deviation $=15), T$-score $($ mean $=50$, standard deviation $=10)$, scaled score $($ mean $=10$, standard deviation $=3), z$-score (mean $=0$, standard deviation $=1$ ), or percentile normed for age. To test cognitive function, the Wechsler Primary Preschool Scale of Intelligence, Fourth Edition (WPPSI-IV); Wechsler Scale of Intelligence for Children, Fifth Edition (WISC-V); or Wechsler Adult Intelligence Scale, Fourth Edition (WAIS-IV) was used. Verbal reasoning was assessed by the Verbal Comprehension Index (VCI) of the Wechsler system. Depending on age, perceptual reasoning and visual spatial reasoning skills were either subsumed by a single Perceptual Reasoning Index (PRI) or separated into the Visual Spatial Index (VSI, the ability to synthesize visual information) and Fluid Reasoning Index (FRI, the ability to identify a solution based on patterns). Working Memory Index (WMI) and Processing Speed Index (PSI) were measured in the Wechsler system if age-appropriate. From the Wechsler Individual Achievement Test, Third Edition (WIAT-III), the Word Reading, Spelling, and Numerical Operations subtests were given to assess academic level.

Speech and language abilities were assessed using the Boston Naming Test (BNT), as well as subscales of the Developmental NEuroPSYchological Assessment, Second Edition (NEPSY-II), and Clinical Evaluation of Language Fundamentals, Fifth Edition (CELF-V). Motor function was assessed by the Purdue Pegboard Test and the Movement Assessment Battery for Children (Movement ABC). For adult subjects, the Movement $\mathrm{ABC}$ was administered and scored for the oldest normed group (16-years-old). To discriminate between perceptual and fine-motor problems, the Beery Buktenika Developmental Test of Visual-Motor Integration, Sixth Edition (VMI-VI) was given. To assess memory, the Rey Complex Figure Test (RCFT) was given for visuospatial organization and visual memory, and the California Verbal Learning Test, Second Edition (CVLT-II) or California Verbal Learning Test, Children's Version (CVLT-C) was given for verbal memory. Processing speed was assessed using the oral response task on the Symbol Digit Modalities Test (SDMT) to reduce the effects of motor dysfunction. Conners Continuous Performance Test, Third Edition (CPT-III) and Conners' Kiddie Continuous Performance Test, Second Edition (K-CPT-II) were given to assess sustained attention. Select subtests from the Delis-Kaplan Executive Function System (D-KEFS) and NEPSY-II were given to assess executive function and social communication, respectively. The Autism Diagnostic Observation Schedule, Second Edition (ADOS-II) was administered to all subjects. To assess sensory, emotional, social, and adaptive function in children, parents completed the Sensory Profile, Second Edition; Behavior Assessment System for Children, Second Edition (BASC-II); Social Responsiveness Scale, Second Edition (SRS-II); and Vineland Adaptive Behavior Scales, Third Edition (Vineland-III).

Whole- exome sequencing. Genomic DNA was isolated from whole-blood samples using the QIAamp DNA Blood Mini Kit (Qiagen) with RNAse A. Each sample was analyzed using NimbleGen SeqCap EZ Exome Library v3.0 64 Mb (300 bp average insert size) to capture the exome. Paired end sequencing was completed using Illumina platforms. Before mapping reads to GRCh37/hg19 (Genome

Reference Consortium), fastq data reads were trimmed of sequencing adapters and filtered for sequence quality using trimGalore against the standard Trueseq adaptor. Reads were aligned to GRCh37/hg19 using BWA, then SAMtools commands were used to clean up paired read information and sort bam files. SAMtools fixmate was used to ensure consistent information display for both reads in a pair then SAMtools sort to order the output file according to genomic sort order. After mapping, the Picard MarkDuplicates command was used to remove or mark PCR duplicates. BEDtools intersect was used to calculate the number of reads, which overlap a target BED file by at least $1 \mathrm{bp}$, yielding an output of the number of ontarget reads. Dividing this number by the total number of mapped, non-duplicate reads yielded the percentage of on-target reads. The GATK function DepthOfCoverage was used to calculate depth of coverage metrics over enrichment targets, 
a

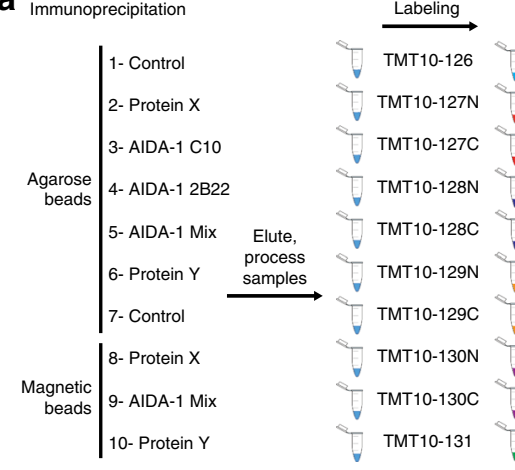

C

\begin{tabular}{lcc}
\multicolumn{3}{c}{ Overlap with AIDA-1 Mix (5) } \\
\hline \multicolumn{1}{c}{ Immunoprecipitation } & Overlap $(\%)$ & $p$ value \\
\hline 2- Protein X & 52.6 & 0.2174 \\
3- AIDA-1 C10 & 21.1 & $<0.0001^{\star \star \star}$ \\
4- AIDA-1 2B22 & 50.0 & $0.0012^{\star \star}$ \\
6- Protein Y & 13.2 & 1.0000 \\
8- Protein X & 7.9 & 0.1086 \\
9- AIDA-1 Mix & 23.7 & $0.0467^{\star}$ \\
10- Protein Y & 7.9 & 0.3979 \\
\hline
\end{tabular}

e

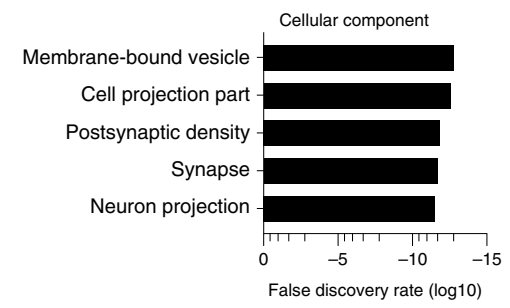

f

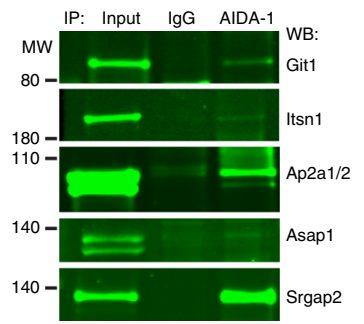

MS identification and quantitation

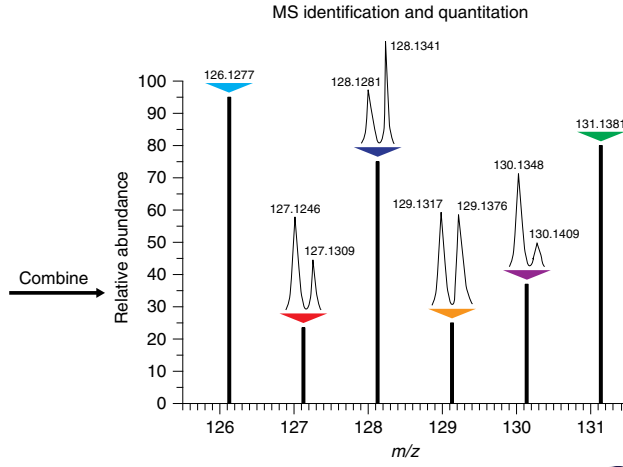

d

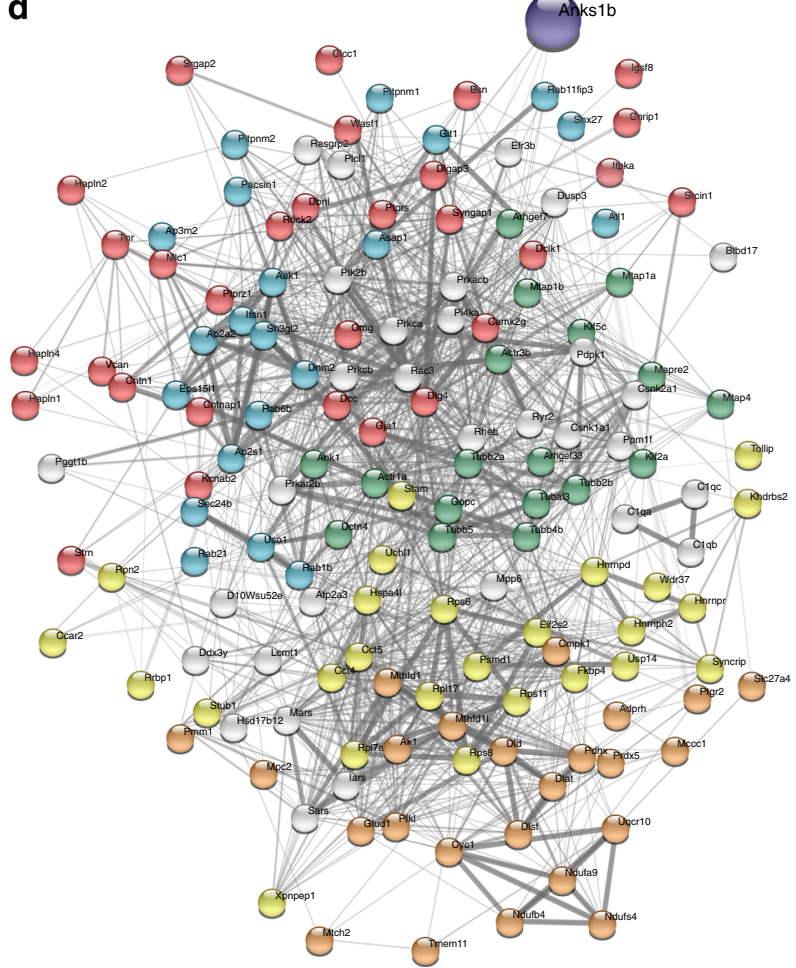

b

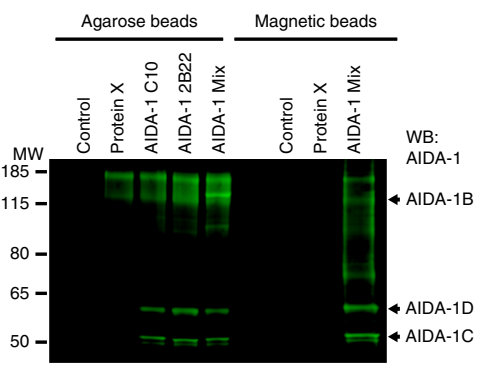

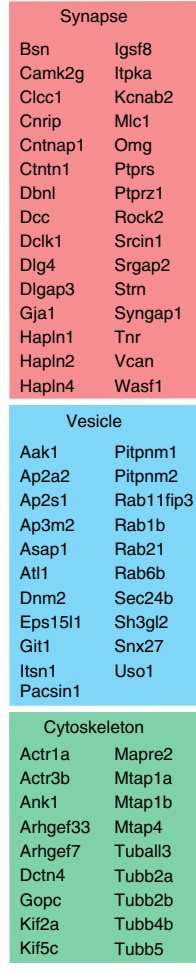

Fig. 4 Identification of the AIDA-1 interactome by mass spectrometry reveals novel cellular roles. a Ten immunoprecipitations (IPs), including four IPs using different AIDA-1 antibodies (C-10, 2B22, or Mix) and beads (agarose or magnetic), two control IPs using mouse IgG, and four control IPs of unrelated synaptic proteins. Samples were differentially labeled using isobaric tags and mixed for MS analysis. Peptides bearing each isobaric tag were simultaneously identified and quantified. b Western blots showing AIDA-1 isoforms immunoprecipitated by AIDA-1 antibody combinations. Only antibody 1A11 (included in AIDA-1 Mix) was able to pull down AIDA-1B. c Overlap of interactors from the AIDA-1 Mix Agarose sample was significant only with interactors from other AIDA-1 IPs by two-sided Fisher's exact test, ${ }^{\star} p<0.05,{ }^{\star \star} p<0.01,{ }^{\star \star \star} p<0.001$. d Depiction of the AIDA-1 interactome prominently featuring components of the synaptic compartment, membrane-bound vesicles, and cytoskeletal projections. (Yellow = ribosome and proteasome; Orange = mitochondria). e Top gene ontology (GO) terms for cellular components enriched in the AIDA-1 interactome. $\mathbf{f}$ Co-IP of novel AIDA-1 interactors from mouse brain lysates. Git1 $=84$ kDa, Itsn1 $=195$ kDa, Ap2a1/2 = 108/104 kDa, Asap1=125 kDa, Srgap2 = 121 kDa

including mean, median, and percent of target bases covered at a depth of at least $30 \times$. To calculate read depth of $A N K S 1 B$ exons in probands, exons within the $A N K S 1 B$ gene were taken as enrichment targets, the counts were gathered for each exon group (1-9, 10-14, and 15-26), and read depths were normalized to the unaffected mother in each trio. To generate the lists of segregated loss-of-function single-nucleotide variants (SNVs) in affected individuals, the list of SNVs for each sample was merged with the ClinVar database of disease annotations. SNVs are listed in Supplementary Data 3 after filtering for High predicted impact on gene expression, then for annotation with specified ClinVar phenotype(s), and finally for segregation with the ANKS1B deletion (present in proband and affected father but not in affected mother).

Tissue samples and antibodies. Hippocampal sections from control postmortem human tissue were obtained from the University of Maryland repositories of the NIH NeuroBioBank. Antibodies used for western blotting were AIDA-1 C-10 (1:1000, Santa Cruz), tubulin (1:1000, Thermo Fisher), PSD95 (1:1000, NeuroMab), GAPDH (1:1000 Cell Signaling Tech), calnexin (1:1000, Genscript), Rab11 (1:1000, Cell Signaling Tech), Git1 (1:1000, Santa Cruz), Itsn1 (1:1000, Santa Cruz), AP2a1/
2 (1:000, Santa Cruz), Asap1 (1:1000, Santa Cruz), and Srgap2 (1:1000, Proteintech). Antibodies for immunocytochemistry were Oct-4 (1:1000, Abcam), Sox2 (1:500, Cell Signaling Tech), MAP-2 (1:1000, EnCor), GluN2B (1:500, Alomone), and GluN2A (1:500, Alomone). Mouse monoclonal antibodies 1A11 and 2B22 were developed and validated in house ${ }^{25}$. Chicken AIDA-1 antibody 5707 was generated by Aves Labs to the peptide sequence GDRLHDDPPQKPPRC at the end of the second SAM domain of AIDA-1.

Human-induced pluripotent stem cells and induced neurons. IPSC generation received ethical approval by the Institutional Review Board (IRB) at Albert Einstein College of Medicine in IRB protocol \#2017-8311 to B.A.J. Informed consent was obtained from all blood donors. Peripheral blood samples were collected from families EIN-1 and EIN-2, and peripheral blood mononuclear CD34 + cells (PBMCs) were used to generate human-induced pluripotent stem cells ${ }^{33}$ (iPSCs) at the Albert Einstein College of Medicine Stem Cell Core. Three iPSC clones from each individual were generated from CD34 + cells using CytoTune-iPS 2.0 Sendai Reprogramming Kit (Invitrogen) following the manufacturer's protocol. Briefly, frozen PBMCs were thawed 2 days before reprogramming (Day -2) and cultured in 
a

\begin{tabular}{lll}
\hline Top diseases and disorders & Annotations & $p$-values \\
\hline Neurological disease & 54 & $5.65 \mathrm{E}-03-2.34 \mathrm{E}-09$ \\
Cancer & 108 & $6.06 \mathrm{E}-03-3.35 \mathrm{E}-08$ \\
Organism injury \& abnormality & 109 & $6.06 \mathrm{E}-03-3.35 \mathrm{E}-08$ \\
Developmental disorder & 33 & $5.33 \mathrm{E}-03-4.22 \mathrm{E}-08$ \\
Gastrointestinal disease & 25 & $5.33 \mathrm{E}-03-4.36 \mathrm{E}-08$ \\
\hline & & \\
\hline Top physiological systems & Annotations & $p$-values \\
\hline Nervous system development \& function & 68 & $5.80 \mathrm{E}-03-1.56 \mathrm{E}-15$ \\
Organismal development & 62 & $5.80 \mathrm{E}-03-1.56 \mathrm{E}-15$ \\
Tissue development & 53 & $5.80 \mathrm{E}-03-1.56 \mathrm{E}-15$ \\
Tissue morphology & 56 & $5.80 \mathrm{E}-03-3.44 \mathrm{E}-12$ \\
Embryonic development & 41 & $5.80 \mathrm{E}-03-1.25 \mathrm{E}-08$ \\
\hline & & \\
\hline Top molecular functions & Annotations & $p$-values \\
\hline Cellular morphology & 63 & $5.80 \mathrm{E}-03-1.56 \mathrm{E}-15$ \\
Cellular assembly \& organization & 62 & $5.80 \mathrm{E}-03-1.56 \mathrm{E}-15$ \\
Cellular development & 47 & $5.80 \mathrm{E}-03-1.56 \mathrm{E}-15$ \\
Cellular function \& maintenance & 60 & $5.80 \mathrm{E}-03-1.56 \mathrm{E}-15$ \\
Cellular growth \& proliferation & 46 & $5.80 \mathrm{E}-03-1.56 \mathrm{E}-15$ \\
\hline
\end{tabular}

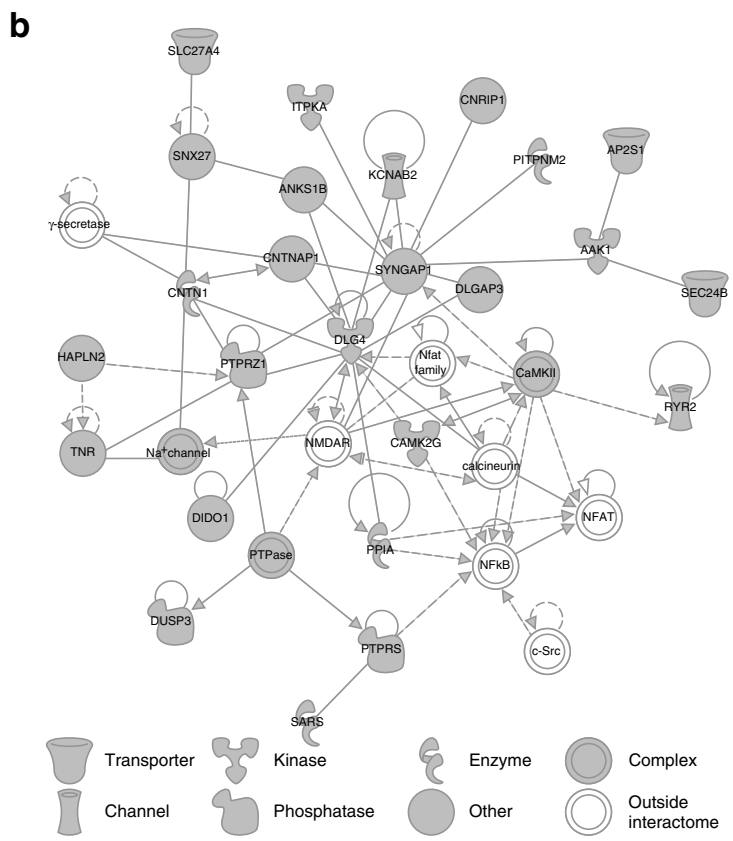

C
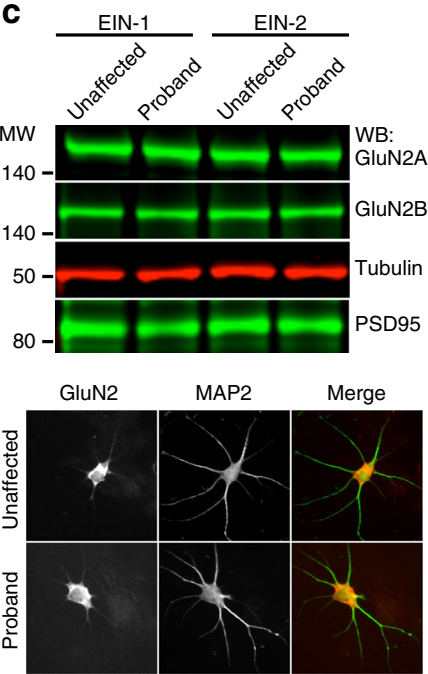

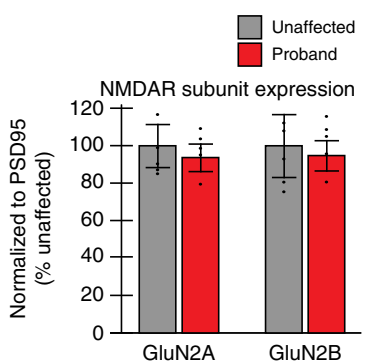

NMDAR surface expression

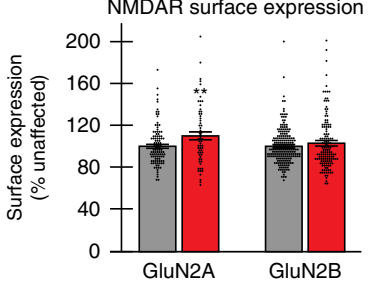

STIF medium. On Day 0, CD34 + cells were flow-sorted by FACSAria II (BD) and transduced with Sendai virus vector of KOS (hKlf4, hOct3/4, and hSox2) at multiplicity of infection (MOI) 5, 5, and 3, respectively, in the presence of Polybrene 4 $\mu \mathrm{g} / \mathrm{mL}$. Three days after transduction, transduced cells were plated on Matrigelcoated 24-well plates in StemSpan SFEM medium (STEMCELL Technologies). Essential 8 (E8) medium (Invitrogen/Thermo Fisher) replaced half of StemSpan SFEM medium on Day 7 and replaced it completely on Day 8. Culture medium was

Fig. 5 Analysis of the AIDA-1 interactome yields functional pathways and mechanisms of disease. a Hierarchical analysis of the most significant diseases and functions in IPA reveals the top disorders, physiological systems, and cellular processes regulated by the AIDA-1 interactome ( $p$ values are given as a range for the diseases and functions annotated in each category). b The top network identified using Ingenuity Pathway Analysis (IPA) revealed known interactors and novel pathways associated with AIDA-1 (network score $=49$, number of focus molecules $=25$ ). Solid lines $=$ direct interaction, dashed lines $=$ indirect interaction, filled arrows $=$ activation, open arrows $=$ translocation, dash $=$ inhibition. $\mathbf{c}$ (Top) Western blot (Family EIN-1 and EIN-2) and quantitation (Family EIN-2) of NMDAR subunits GluN2A and GluN2B in iPSC-derived neurons show no changes in probands ( $10 \mu$ g lysate). $N=3$ biological replicates. (Bottom) Sample images (GluN2B) and quantitation of GluN2A and GluN2B surface expression in neurons from proband EIN-2-1 and unaffected mother EIN-2$M$ reveal a significant increase in GluN2A, but no change in GluN2B. $N=3$ biological replicates based on 60-99 neurons. Scale bar $=10 \mu \mathrm{m}$. Bar graphs show mean \pm SEM, two-sided Student's $t$-test, ${ }^{\star} p<0.05$, ${ }^{\star *} p<0.01$

changed every 2 days from Day 2 to Day 7 and changed daily from Day 8. The iPSlike clones were picked and passaged by mechanical dissection from Day 21 to Day 28. FACS analysis of pluripotency markers SSEA3, SSEA4, TRA-1-60, and TRA-181; in vitro differentiation and immunohistochemical detection of germ-layer markers $\alpha$-fetoprotein, $\alpha$-smooth muscle actin, and $\beta$-III tubulin; RT-PCR assay for virus gene integration; and karyotyping were performed on each iPSC clone to ensure the quality of integration-free iPSCs. Clones were maintained in E8 as described by the manufacturer with occasional removal of differentiated cells. IPSC identity was periodically confirmed by immunocytochemistry using pluripotency markers Oct-4 and Sox-2.

Induced neurons (iNs) were generated by forced expression of the human neuronal transcription factor neurogenin-2 (NGN2) in iPSCs and growing cells in the presence of neurotrophic factors and rat astrocytes to facilitate maturation into excitatory neurons ${ }^{34}$. Briefly, iPSCs were treated with trypLE, dissociated for $5 \mathrm{~min}$, and plated on Matrigel-coated plates in E8 medium containing ROCK inhibitor $1 \mu \mathrm{M}$ (H-1152 dihydrocholoride, Santa Cruz). Cells were plated on Matrigel-coated 24-well plates for western blotting, and on Matrigel-coated coverslips for immunocytochemistry. On Day 2, cells were infected with lentivirus (NGN2 and rtTA, Addgene) in E8. On Day 3, media was replaced with DMEM/F12 (Thermo Fisher) containing N-2 1\% (Invitrogen), NEAA 1\% (Thermo Fisher), brain-derived neurotrophic factor (BDNF) $10 \mathrm{ng} / \mathrm{mL}$ (GerminiBio), NT-3 $10 \mathrm{ng} / \mathrm{mL}$

(GerminiBio), laminin $0.2 \mu \mathrm{g} / \mathrm{mL}$ (Santa Cruz), and doxycycline $4 \mu \mathrm{g} / \mathrm{mL}$ (Santa Cruz). On Day 4, cells were selected in puromycin $1 \mu \mathrm{g} / \mathrm{mL}$ for $24 \mathrm{~h}$. On Day 5 , rat astrocytes were added in DMEM/F12 containing BDNF, NT-3, and 0.5 $\mu \mathrm{M}$ Ara-C (Santa Cruz). On Day 6, medium was replaced with Neurobasal medium (Thermo Fisher) supplemented with B-27 (Thermo Fisher), GlutaMAX (Thermo Fisher), BDNF, NT-3, and doxycycline. On Day 10, 2.5\% fetal bovine serum was added to the medium. On Day 19, iNs were lysed or fixed for analysis.

Imaging. For histological analysis, adult mice at 16 weeks of age were perfused with $4 \%$ PFA. Brains were collected and medial sagittal brain sections ( $40 \mu \mathrm{m}$ thick) were prepared and processed for cresyl violet-based Nissl staining. Images were acquired on a Zeiss Axio Scan.Z1 slide scanner with a 5x objective. Areas were quantified using ZEN software (blue edition). Immunofluorescence of iPSCderived neurons was performed using standard procedures and imaged on a Zeiss AxioObserver microscope at 20x. To measure surface NMDAR subunits, Day 28 induced neurons were fixed and immunostained with GluN2A or GluN2B antibodies, then permeabilized with $0.01 \%$ Triton X-100 and immunostained with the neuronal and dendritic marker MAP2. All measurements were obtained and quantified by an experimenter blind to genotype.

\section{Reverse transcription quantitative polymerase chain reaction (RT-qPCR).}

RNA was isolated from iPSC-derived neuronal cultures and from Nestin-Het and Nestin-WT mice using the RNeasy Plus Mini Kit (Qiagen). Primers were designed to ANKS1B transcripts for Exon 4 (forward: 5'-GCCCTACACTGTGCAGCTCA ATA-3', reverse: 5'-GGGTCAGTGAGCTCTTCTAGGAG-3'), Exon 13 (forward: 5'-ACCATACCATTGTTGGCACAAG-3', reverse: 5' -ACAAATCCCCCTGCG TTCAT-3'), and Exon 20 (forward: 5'-ACCTCCGAATGAAGCCACAG-3', reverse: $5^{\prime}$-GCTTTGTAATCACACGACTGGA-3'). In all experiments, the neuronal marker MAP2 (forward: 5'-TTGGTGCCGAGTGAGAAGA-3', reverse: 5'GTCTGGCAGTGGTTGGTTAA- $\left.3^{\prime}\right)^{70}$ was used as the housekeeping gene.

Anks1b Nestin-Het mouse model. To generate the Anks1b heterozygous conditional knockout line, male mice from the transgenic B6.Cg- $\mathrm{Tg}($ Nes-cre $) 1 \mathrm{Kln} / \mathrm{J}$ line 
(Nestin-Cre) were purchased from Jackson Laboratories (\#003771) and crossed to female mice from the Anks 1 fllox $^{\text {flox }}$ line previously generated ${ }^{25}$. The Nestin-Het mouse line was made congenic by backcrossing to C57BL/6J mice (Jackson Laboratories, \#000664) for at least ten generations prior to behavioral testing. For all western blotting, RT-qPCR, gross and histological morphometry, and behavioral assays, the Nestin-Cre;Anks $1 b^{\text {wt fllox }}$ mice generated from this colony were used as mutants (Nestin-Het) while Nestin-Cre;Anks $1 b^{w t / w t}$ mice (Nestin-WT) were used as controls.

Animal behavioral assays. All experiments complied with all relevant ethical regulations for animal testing and research, and were approved by the Albert Einstein College of Medicine Institutional Animal Care and Use Committee (IACUC). Mice were housed and handled at the Albert Einstein College of Medicine and behavioral phenotyping was performed as five independent cohorts in the Albert Einstein College of Medicine Animal Behavior Core under the supervision of Dr. Maria Gulinello. In the Behavioral Spectrometer ${ }^{38}$, mice were recorded for $9 \mathrm{~min}$ in an open field with a center area of $18.0 \times 18.0 \mathrm{~cm}$. Elevated plus maze $(5 \mathrm{~min})$, forced swim test $\left(9 \mathrm{~min}\right.$ in $25^{\circ} \mathrm{C}$ water bath), and three-chamber test $(5$ min with ovariectomized C57BL/6J mouse) were performed using standard procedures $^{71}$. Social preference was calculated using the following formula: social preference $=($ social sniffing time $) /($ social sniffing time + object sniffing time $) \times$ $100 \%$. Acoustic startle reflex and prepulse inhibition of the startle response were assayed ${ }^{72}$ in a single session for each mouse using randomized, interleaved trials (five each) for acoustic startle response (115 dB) and prepulse inhibition (PPI) Short-delay PPI trials were conducted with an $81-\mathrm{dB}$ prepulse $40 \mathrm{~ms}$ before the 115 - $\mathrm{dB}$ target stimulus, while long-delay PPI was tested with a 200 -ms interstimulus interval. Adhesive removal tests were performed ${ }^{41}$ as a single trial by applying a $0.5 \times 4.0 \mathrm{~cm}$ strip of Micropore (3M) medical adhesive to the left forepaw after a 30 -min habituation to an empty mouse cage. Balance beam assay for motor coordination was performed ${ }^{73}$ on a $1.2 \mathrm{~cm}$ round wooden beam after pre-training. The novel object placement test was performed ${ }^{74}$ using a 5 -min training phase, 4-min testing phase, and short (40 $\mathrm{min}$ ) or long (90 min) retention intervals. During the testing phase, preference for the new placement was calculated using the following formula: preference for new $=($ time exploring new placement)/(time exploring new placement + time exploring old placement $) \times 100 \%$.

All behavioral testing in adult mice aged 3-4 months and in pups aged P8-P16 was performed with experimenter blind to genotype. Feeding, mixed-genotype group housing, light-dark cycles, and time of testing were controlled across all five cohorts $^{75}$. Sample sizes were estimated in JMP (version 14, SAS) to be of sufficient power to show effects independently in either sex if main effect of genotype-sex interaction $(\alpha=0.05)$ were detected in two-way ANOVA (Supplementary Data 5). For all behavioral tests, post hoc two-sided Student's $t$-test was performed for tests in which genotype was a significant main effect in two-way ANOVA. For the threechamber test, likelihood ratio tests were additionally performed for effects of Genotype and Sex on passing ( $>50 \%$ social preference) or failing $(<50 \%$ social preference). Post hoc contingency testing included chi-squared analyses and twosided Fisher's exact test. To assess differences between social and object sniffing times, repeated-measures ANOVA was also performed using a within-subjects design with Social time and Object time as responses and Genotype and Sex as factors. Sniffing times were separated by Social/Object and genotype, and post hoc testing was conducted using Tukey Kramer Honest Significant Difference (HSD) test for multiple comparisons. Since no main effect of sex or genotype-sex interaction was observed in any behavioral test, males and females of each genotype were combined for all post hoc testing, and samples of $N>20$ were used to satisfy the Central Limit Theorem in lieu of testing for normality ${ }^{75}$. Power analysis and least significant number (LSN) were calculated in JMP for post hoc two-sided Student's $t$-tests on significant effects from two-way ANOVA analyses. Developmental milestone testing was conducted ${ }^{42}$ at P10, P12, P14, and P16: tests included homing time to nesting material in the home cage, negative geotaxis righting time at $45^{\circ}$, and acoustic startle reflex scoring $(0=$ none, $1=$ ear twitch, 2 $=$ head twitch, 3 = full body startle). ANOVA was performed to detect main effects and interactions of age, genotype, and sex: since age was an expected main effect and did not interact with any other factors, no post hoc testing was conducted. Ultrasonic vocalizations from maternally isolated pups were recorded at P8 and P10 using Avisoft Bioacoustics software for 6 min and analyzed using MUPET, an unsupervised machine-learning-based algorithm that analyzes vocalization parameters, classifies syllables into repertoires, and compares between test groups to generate a similarity score ${ }^{76}$.

Multiplexed large-scale immunoprecipitations. To perform immunoprecipitations (IPs), we incubated $300 \mu \mathrm{g}$ of antibodies with $200 \mu \mathrm{l}$ of agarose (Protein G Sepharose) beads for $1 \mathrm{~h}$ at $4{ }^{\circ} \mathrm{C}$ in PBS, washed $2 \mathrm{x}$ with $0.2 \mathrm{M}$ triethanolamine $\mathrm{pH}$ 8.2 (TEA), and then crosslinked using freshly made $30 \mathrm{mM}$ dimethyl pimelimidate (DMP) in TEA for $25 \mathrm{~min}$ at room temperature (RT). Beads were washed for $5 \mathrm{~min}$ with TEA, crosslinked again using a fresh DMP solution, washed $3 \mathrm{x}$ with TEA, and quenched using two 10 -min washes of $100 \mathrm{mM}$ ethanolamine. Unbound antibodies were stripped off beads $3 \mathrm{x}$ using $100 \mathrm{mM}$ glycine $\mathrm{pH} 3.1$ for $5 \mathrm{~min}$, and beads were then washed $2 \mathrm{x}$ with $\mathrm{PBS}$ and stored in $\mathrm{PBS} / \mathrm{NaN}_{3}$. For crosslinking antibodies to magnetic beads, we coupled $90 \mu \mathrm{g}$ of antibodies to $6 \mathrm{mg}$ of Epoxy Dynabeads (Dynal) according to the manufacturer instructions. Antibodies used were: mouse
IgG, AIDA-1 C-10 (Santa Cruz), AIDA-1 2B22, or AIDA-1 Mix (C-10 + 1A11 + 2B22). Each individual IP was split into two reactions: one IP consisted of $40 \mu \mathrm{g}$ coupled antibodies incubated with $5 \mathrm{mg}$ of total mouse brains (whole brain, including olfactory bulb, cortices, midbrain, and cerebellum dissected from 3month-old C57BL/6J mice) lysed in a gentle dodecyl- $\beta$-maltoside buffer (DBM: $10 \mathrm{mM}$ HEPES pH 7.4, $190 \mathrm{mM} \mathrm{NaCl}, 10 \mathrm{mM} \mathrm{KCl}, 1 \mathrm{mM}$ EGTA, $1 \%$ dodecyl- $\beta$ maltoside), and the second IP consisted of $40 \mu \mathrm{g}$ incubated with $5 \mathrm{mg}$ of lysate in a harsher RIPA buffer $(25 \mathrm{mM}$ Tris $\mathrm{pH} 7.4,300 \mathrm{mM} \mathrm{NaCl}, 1 \%$ Triton X-100, $0.5 \%$ deoxycholate, $0.1 \%$ SDS, $2 \mathrm{mM}$ EDTA). This approach allowed for the capture of detergent-sensitive interactions (DBM) and additional interactions in detergentresistant synaptic and nuclear structures (RIPA). We incubated IPs for $2 \mathrm{~h}$ at RT with rocking, added two volumes of either $10 \mathrm{mM}$ HEPES (DBM lysates) or $25 \mathrm{mM}$ Tris (RIPA lysates) to dilute salts and detergents, and incubated overnight at $4{ }^{\circ} \mathrm{C}$. Beads were washed $5 \mathrm{x}$ with TBS/Tween-20, DBM and RIPA IPs for each antibody sample were mixed together, and bound proteins were eluted in $2 \mathrm{x}$ non-reducing Laemmli sample buffer at $70^{\circ} \mathrm{C}$ for $30 \mathrm{~min}$.

Tandem mass tag (TMT) labeling of peptides. Ten micrograms of each immunoprecipitation sample was electrophoresed briefly (dye front $5 \mathrm{~mm}$ ) into a $15 \%$ sodium dodecyl sulfate polyacrylamide gel electrophoresis gel. The gel was washed $3 \mathrm{x}$ in $\mathrm{ddH}_{2} \mathrm{O}$ for 15 min each and visualized by staining overnight with GelCode $^{\circledast}$ Coomassie blue reagent (Pierce). Stacked protein bands were excised from the gel, reduced with dithiothreitol, and alkylated with iodoacetamide. In-gel digestion was performed using $5 \mathrm{ng} / \mu \mathrm{L}$ mass spectrometry-grade trypsin (Trypsin Gold, Promega) in $50 \mathrm{mM} \mathrm{NH}_{4} \mathrm{HCO}_{3}$ digestion buffer. The resulting peptides were desalted using a Stage Tip manually packed with Empora C18 High-Performance Extraction Disks $(3 \mathrm{M})^{77}$ and eluted peptide solutions were dried under vacuum. Peptides were then resuspended in $18 \mu \mathrm{L}$ acetonitrile, and $57 \mu \mathrm{L}$ of $0.2 \mathrm{M}$ HEPES $\mathrm{pH} 8.5$ was added to each sample. TMT10-plex amine reactive reagents (Thermo Fisher, $5 \mathrm{mg}$ per vial) were resuspended in $1024 \mu \mathrm{L}$ anhydrous acetonitrile and $25 \mu \mathrm{L}$ of reagent was added to each sample (TMT label: peptide $[w / w]=12: 1$ ) and mixed briefly by vortexing. The mixture was incubated at RT for $1 \mathrm{~h}$, quenched by the addition of $10 \mu \mathrm{L} 5 \%$ hydroxylamine for $15 \mathrm{~min}$, and acidified by the addition of $10 \mu \mathrm{L} 10 \%$ formic acid. A $5-\mu \mathrm{L}$ aliquot from each reaction was desalted on a StageTip, analyzed by liquid-chromatography-tandem mass spectrometry (LC-MS/MS) with a Q Exactive Orbitrap HF (high field), and the resulting spectra searched with MaxQuant using its corresponding TMT label as variable modifications on $\mathrm{N}$-terminus and lysine. The percentage of peptides with either $\mathrm{N}$ terminal or lysine TMT labels was calculated, indicating the labeling efficiency for each channel. Labeling efficiency was $96 \%$ or greater for each channel. To ensure that equal amounts of labeled peptides from each channel were mixed together, a two-step mixing strategy was employed: in the first step, an identical $\sim 1 \mu \mathrm{L}$ volume of peptides from each channel was mixed and analyzed, and the value of the median ratio (median of the ratios of all peptide intensities of one channel over their corresponding peptide average intensities of all channels) for each channel is determined as the correction factor. In the second step, the rest of the peptides were mixed by adjusting their volume using the correction factors. In this way, median ratios ranging from 0.97 to 1.02 were achieved as previously reported ${ }^{78}$. The final mixture of reaction products from ten TMT channels were desalted on a Sep-Pak tC18 $1 \mathrm{~mL}$ Vac Cartridge (Waters, \#WAT03820). Eluted peptides were dried by vacuum centrifugation and stored at $-20^{\circ} \mathrm{C}$.

Liquid-chromatography-tandem mass spectrometry. Online chromatography was performed with a Thermo Easy nLC 1000 ultrahigh-pressure UPLC system (Thermo Fisher) coupled online to a Q Exactive HF with a NanoFlex source (Thermo Fisher). Analytical columns $(\sim 30 \mathrm{~cm}$ long and $75 \mu \mathrm{m}$ inner diameter) were packed in house with ReproSil-Pur C18 AQ $3 \mu \mathrm{M}$ reversed-phase resin (Dr Maisch $\mathrm{GmbH}$, Ammerbuch-Entringen). The analytical column was placed in a column heater (Sonation $\mathrm{GmbH}$, Biberach) regulated to a temperature of $45^{\circ} \mathrm{C}$. The TMT peptide mixture was loaded onto the analytical column with buffer $\mathrm{A}$ $(0.1 \%$ formic acid $)$ at a maximum back-pressure of 300 bar. Peptides were eluted with a 2 -step gradient of 3 to $40 \%$ buffer B ( $100 \% \mathrm{ACN}$ and $0.1 \%$ formic acid) in $180 \mathrm{~min}$ and 40 to $90 \% \mathrm{~B}$ in $20 \mathrm{~min}$, at a flow rate of $250 \mathrm{~nL} / \mathrm{min}$ over $200 \mathrm{~min}$ using a 1D online LC-MS2 data-dependent analysis (DDA) method as follows: MS data were acquired using a data-dependent top-10 method, dynamically choosing the most abundant not-yet-sequenced precursor ions from the survey scans (300-1750 Th). Peptide fragmentation was performed via higher energy collisional dissociation (HCD) with a target value of $1 \times 10^{5}$ ions determined with predictive automatic gain control. Isolation of precursors was performed with a window of 1 Th. Survey scans were acquired at a resolution of 120,000 at $\mathrm{m} / \mathrm{z} 200$. Resolution for HCD spectra was set to 60,000 at $\mathrm{m} / \mathrm{z} 200$ with a maximum ion injection time of $128 \mathrm{~ms}$. The normalized collision energy was 35 . The underfill ratio specifying the minimum percentage of the target ion value likely to be reached at the maximum fill time was defined as $0.1 \%$. Precursor ions with single, unassigned, or seven and higher charge states were excluded from fragmentation selection. Dynamic exclusion time was set at $30 \mathrm{~s}$. Each of the TMT 10-plex samples was analyzed in triplicate.

All data were analyzed with the MaxQuant proteomics data analysis workflow (version 1.5.5.1) with the Andromeda search engine ${ }^{79,80}$. The type of the group specific analysis was set to Reporter ion MS2 with 10-plex TMT as isobaric labels 
for Q Exactive High Field MS2 data. Reporter ion mass tolerance was set to 0.01 $\mathrm{Da}$, with activated Precursor Intensity Fraction (PIF) value set at 0.75 . False discovery rate was set to $1 \%$ for protein, peptide spectrum match, and site decoy fraction levels. Peptides were required to have a minimum length of eight amino acids and a maximum mass of $4600 \mathrm{Da}$. MaxQuant was used to score fragmentation scans for identification based on a search with an allowed mass deviation of the precursor ion of up to $4.5 \mathrm{ppm}$ after time-dependent mass calibration. The allowed fragment mass deviation was $20 \mathrm{ppm}$. MS2 spectra were used by Andromeda within MaxQuant to search the Uniprot mouse database (01092015; 16,699 entries) combined with 262 common contaminants. Enzyme specificity was set as C-terminal to arginine and lysine, and a maximum of two missed cleavages were allowed. Carbamidomethylation of cysteine was set as a fixed modification and $\mathrm{N}$-terminal protein acetylation, deamidated $(\mathrm{N}, \mathrm{Q})$ and oxidation (M) as variable modifications. The reporter ion intensities were defined as intensities multiplied by injection time (to obtain the total signal) for each isobaric labeling channel summed over all MS/MS spectra matching to the protein group as previously validated $^{80}$. Following MaxQuant analysis, the protein and peptide.txt files were imported into Perseus (version 1.5.6.0) software, which was used for statistical analysis of all the proteins identified.

Identification and analysis of the AIDA-1 interactome. Enrichment for proteins in each 10-plex IP sample was calculated by dividing the relative abundance in the IP by the relative abundance in the appropriate control mouse IgG bead sample (agarose or magnetic) (Supplementary Data 6a). In the agarose bead experiments, analysis of all abundance values showed that the top $5 \%$ were above $~ 1.4$-fold enrichment, while the cutoff for the top $5 \%$ in magnetic bead experiments was $\sim 3.0$-fold. The appropriate cutoff was applied to each IP sample to obtain an enriched population of 255 proteins that were $\geq 1$.4-fold enriched in any agarose bead experiment or $\geq 3$.0-fold enriched in any magnetic bead experiment. Only proteins with three or more peptides identified were included in the analysis, to obtain a reliable mean relative abundance for each protein. The list of proteins enriched in any AIDA-1 IP sample (defined as the AIDA-1 interactome, Supplementary Data 6b) was used as the input for multiple proteins in StringDB under the species Mus musculus. Raw data for gene ontology (GO) enrichments are given in Supplementary Data 7, and the protein-protein interaction image (Fig. 4d) was generated by setting the minimum interaction score to 0.150 , hiding disconnected network nodes, and displaying connections by strength of supporting data ${ }^{44}$. For functional annotation and network construction in Ingenuity Pathway Analysis (QIAGEN Bioinformatics, version 01-13), the AIDA-1 interactome (Supplementary Data 6b) was used as input for a Core Analysis using the following default settings: Expression Analysis; General Settings = Ingenuity Knowledge Base (Genes Only), Direct and Indirect Relationships; Networks = Interaction networks, Include endogenous chemicals; Node Types = All; Data Sources = All; Confidence $=$ Experimentally Observed; Species $=$ All; Tissues \& Cell Lines $=$ All; Mutations $=$ All. The top-scoring network from this analysis (Network 1) is depicted in Fig. 5a. Raw data obtained from the analysis is included as supplementary Supplementary Data 8.

Statistical analysis. Statistical tests were performed in JMP (version 14, SAS) as in Supplementary Data 4. For AIDA-1 expression by western blot (Fig. 1g and Supplementary Fig. 2c), two-way ANOVA was performed to probe for main effect of Genotype (Unaffected/WT or Proband/Het), Band (AIDA-1 isoform), and Genotype-Band interaction. Although no isoform-specific effects of genotype were detected, two-sided Student's $t$-tests were performed as indicated. For RT-qPCR expression, two-way ANOVA was performed for main effect of Genotype (Unaffected/WT or Proband/Het), Target (Exon number), and Genotype-Target interaction (Fig. 1h and Supplementary Fig. 2d). Although no exon-specific effects of genotype were detected, one-sided Student's $t$-tests were performed as indicated. For gross morphological analysis (Fig. 2d), sex differences were expected and two-sided Student's $t$-tests were performed independently for each sex. For histological analysis of brain regions, two-way ANOVA showed no effects of Genotype, Sex, or Genotype-Sex interaction. Sexes were combined for two-sided Student's $t$-tests. To analyze specificity of the AIDA-1 interactome, two-sided Fisher's exact test was performed on the number of proteins enriched in each sample, with the AIDA-1 Mix agarose bead sample as a reference for overlap and the total number of proteins enriched in any sample as the statistical background.

Reporting summary. Further information on research design is available in the Nature Research Reporting Summary linked to this article.

\section{Data availability}

All raw data and the datasets generated during and/or analyzed during the current study are available from the corresponding author on reasonable request.

Received: 21 December 2018 Accepted: 13 July 2019

Published online: 06 August 2019

\section{References}

1. Geschwind, D. H. Genetics of autism spectrum disorders. Trends Cogn. Sci. 15 409-416 (2011).

2. Weiner, D. J. et al. Polygenic transmission disequilibrium confirms that common and rare variation act additively to create risk for autism spectrum disorders. Nat. Genet. 49, 978-985 (2017).

3. Krumm, N. et al. Excess of rare, inherited truncating mutations in autism. Nat. Genet. 47, 582-588 (2015).

4. Marshall, C. R. \& Scherer, S. W. Detection and characterization of copy number variation in autism spectrum disorder. Methods Mol. Biol. 838, 115-135 (2012).

5. Hu, V. W., Addington, A. \& Hyman, A. Novel autism subtype-dependent genetic variants are revealed by quantitative trait and subphenotype association analyses of published GWAS data. PLoS ONE 6, e19067 (2011).

6. Uddin, M. et al. Brain-expressed exons under purifying selection are enriched for de novo mutations in autism spectrum disorder. Nat. Genet. 46, 742-747 (2014).

7. Pinto, D. et al. Convergence of genes and cellular pathways dysregulated in autism spectrum disorders. Am. J. Hum. Genet. 94, 677-694 (2014).

8. Liu, L. et al. DAWN: a framework to identify autism genes and subnetworks using gene expression and genetics. Mol. Autism 5, 22 (2014).

9. Li, J. et al. Integrated systems analysis reveals a molecular network underlying autism spectrum disorders. Mol. Syst. Biol. 10, 774 (2014).

10. Meehan, T. F. et al. Autism candidate genes via mouse phenomics. J. Biomed. Inf. 44(Suppl 1), S5-S11 (2011).

11. Umehara, H. et al. Calcium signaling pathway is associated with the long-term clinical response to selective serotonin reuptake inhibitors (SSRI) and SSRI with antipsychotics in patients with obsessive-compulsive disorder. PLoS ONE 11, e0157232 (2016).

12. Grunblatt, E. et al. High resolution chromosomal microarray analysis in paediatric obsessive-compulsive disorder. BMC Med Genom. 10, 68 (2017).

13. Garriock, H. A. et al. A genomewide association study of citalopram response in major depressive disorder. Biol. Psychiatry 67, 133-138 (2010).

14. Lydall, G. J. et al. Confirmation of prior evidence of genetic susceptibility to alcoholism in a genome-wide association study of comorbid alcoholism and bipolar disorder. Psychiatr. Genet. 21, 294-306 (2011).

15. McClay, J. L. et al. Genome-wide pharmacogenomic analysis of response to treatment with antipsychotics. Mol. Psychiatry 16, 76-85 (2011).

16. Ren, H. et al. Unravelling genes and pathways implicated in working memory of schizophrenia in Han Chinese. Int J. Mol. Sci. 16, 2145-2161 (2015).

17. Kang, S. G., Chee, I. S., Lee, K. \& Lee, J. rs7968606 polymorphism of ANKS1B is associated with improvement in the PANSS general score of schizophrenia caused by amisulpride. Hum. Psychopharmacol. 32, e2562 (2017).

18. Chang, $\mathrm{X}$. et al. Common and rare genetic risk factors converge in protein interaction networks underlying schizophrenia. Front. Genet. 9, 434 (2018).

19. Ghersi, E., Noviello, C. \& D'Adamio, L. Amyloid-beta protein precursor (AbetaPP) intracellular domain-associated protein-1 proteins bind to AbetaPP and modulate its processing in an isoform-specific manner. J. Biol. Chem. 279, 49105-49112 (2004)

20. Jacob, A. L., Jordan, B. A. \& Weinberg, R. J. Organization of amyloid-beta protein precursor intracellular domain-associated protein-1 in the rat brain. J. Comp. Neurol. 518, 3221-3236 (2010).

21. Jordan, B. A. et al. Identification and verification of novel rodent postsynaptic density proteins. Mol. Cell Proteom. 3, 857-871 (2004).

22. Lowenthal, M. S., Markey, S. P. \& Dosemeci, A. Quantitative mass spectrometry measurements reveal stoichiometry of principal postsynaptic density proteins. J. Proteome Res. 14, 2528-2538 (2015).

23. Jordan, B. A., Fernholz, B. D., Khatri, L. \& Ziff, E. B. Activity-dependent AIDA-1 nuclear signaling regulates nucleolar numbers and protein synthesis in neurons. Nat. Neurosci. 10, 427-435 (2007).

24. $\mathrm{Xu}, \mathrm{H}$. \& Hebert, M. D. A novel EB-1/AIDA-1 isoform, AIDA-1c, interacts with the Cajal body protein coilin. BMC Cell Biol. 6, 23 (2005).

25. Tindi, J. O. et al. ANKS1B gene product AIDA-1 controls hippocampal synaptic transmission by regulating GluN2B subunit localization. J. Neurosci. 35, 8986-8996 (2015).

26. Firth, H. V. et al. DECIPHER: database of chromosomal imbalance and phenotype in humans using ensembl resources. Am. J. Hum. Genet. 84, 524-533 (2009).

27. Sobreira, N., Schiettecatte, F., Valle, D. \& Hamosh, A. GeneMatcher: a matching tool for connecting investigators with an interest in the same gene. Hum. Mutat. 36, 928-930 (2015).

28. Landrum, M. J. et al. ClinVar: improving access to variant interpretations and supporting evidence. Nucl. Acids Res. 46, D1062-D1067 (2018).

29. Huang, N., Lee, I., Marcotte, E. M. \& Hurles, M. E. Characterising and predicting haploinsufficiency in the human genome. PLoS Genet. 6, e1001154 (2010).

30. Lek, M. et al. Analysis of protein-coding genetic variation in 60,706 humans. Nature 536, 285-291 (2016). 
31. Samocha, K. E. et al. Regional missense constraint improves variant deleteriousness prediction. Preprint at https://www.biorxiv.org/content/ 10.1101/148353v1 (2017).

32. Cunningham, F. et al. Ensembl 2019. Nucl. Acids Res. 47, D745-D751 (2018).

33. Okita, K., Hong, H., Takahashi, K. \& Yamanaka, S. Generation of mouseinduced pluripotent stem cells with plasmid vectors. Nat. Protoc. 5, 418-428 (2010).

34. Zhang, Y. et al. Rapid single-step induction of functional neurons from human pluripotent stem cells. Neuron 78, 785-798 (2013).

35. Teng, S., Yang, J. Y. \& Wang, L. Genome-wide prediction and analysis of human tissue-selective genes using microarray expression data. BMC Med. Genom. 6(Suppl 1), S10 (2013).

36. Liang, H., Hippenmeyer, S. \& Ghashghaei, H. T. A Nestin-cre transgenic mouse is insufficient for recombination in early embryonic neural progenitors. Biol. Open 1, 1200-1203 (2012).

37. Giusti, S. A. et al. Behavioral phenotyping of Nestin-Cre mice: implications for genetic mouse models of psychiatric disorders. J. Psychiatr. Res. 55, 87-95 (2014).

38. Brodkin, J. et al. Validation and implementation of a novel high-throughput behavioral phenotyping instrument for mice. J. Neurosci. Methods 224, 48-57 (2014).

39. Bannerman, D. M. et al. Hippocampal synaptic plasticity, spatial memory and anxiety. Nat. Rev. Neurosci. 15, 181-192 (2014).

40. Silverman, J. L., Yang, M., Lord, C. \& Crawley, J. N. Behavioural phenotyping assays for mouse models of autism. Nat. Rev. Neurosci. 11, 490-502 (2010).

41. Bouet, V. et al. The adhesive removal test: a sensitive method to assess sensorimotor deficits in mice. Nat. Protoc. 4, 1560-1564 (2009).

42. Scattoni, M. L., Gandhy, S. U., Ricceri, L. \& Crawley, J. N. Unusual repertoire of vocalizations in the BTBR T+tf/J mouse model of autism. PLoS ONE 3, e3067 (2008).

43. Thompson, A. et al. Tandem mass tags: a novel quantification strategy for comparative analysis of complex protein mixtures by MS/MS. Anal. Chem. 75, 1895-1904 (2003).

44. Szklarczyk, D. et al. STRING v10: protein-protein interaction networks, integrated over the tree of life. Nucl. Acids Res. 43, D447-D452 (2015).

45. Gurd, J. W., Jones, L. R., Mahler, H. R. \& Moore, W. J. Isolation and partial characterization of rat brain synaptic plasma membranes. J. Neurochem. 22, 281-290 (1974).

46. Welz, T., Wellbourne-Wood, J. \& Kerkhoff, E. Orchestration of cell surface proteins by Rab11. Trends Cell Biol. 24, 407-415 (2014).

47. Valdes, J. L. et al. Sorting nexin 27 protein regulates trafficking of a p21activated kinase (PAK) interacting exchange factor (beta-Pix)-G proteincoupled receptor kinase interacting protein (GIT) complex via a PDZ domain interaction. J. Biol. Chem. 286, 39403-39416 (2011).

48. Pechstein, A. et al. Regulation of synaptic vesicle recycling by complex formation between intersectin 1 and the clathrin adaptor complex AP2. Proc. Natl Acad. Sci. 107, 4206-4211 (2010).

49. Inoue, H., Ha, V. L., Prekeris, R. \& Randazzo, P. A. Arf GTPase-activating protein ASAP1 interacts with Rab11 effector FIP3 and regulates pericentrosomal localization of transferrin receptor-positive recycling endosome. Mol. Biol. Cell 19, 4224-4237 (2008).

50. Fossati, M. et al. SRGAP2 and its human-specific paralog co-regulate the development of excitatory and inhibitory synapses. Neuron 91, 356-369 (2016).

51. Wilkinson, B., Li, J. \& Coba, M. P. Synaptic GAP and GEF complexes cluster proteins essential for GTP signaling. Sci. Rep. 7, 5272 (2017).

52. Cai, L., Loo, L. S., Atlashkin, V., Hanson, B. J. \& Hong, W. Deficiency of sorting nexin 27 (SNX27) leads to growth retardation and elevated levels of N-methyl-D-aspartate receptor 2C (NR2C). Mol. Cell Biol. 31, 1734-1747 (2011).

53. Habela, C. W., Song, H. \& Ming, G. L. Modeling synaptogenesis in schizophrenia and autism using human iPSC derived neurons. Mol. Cell Neurosci. 73, 52-62 (2016).

54. Webb, A. et al. RNA sequencing of transcriptomes in human brain regions: protein-coding and non-coding RNAs, isoforms and alleles. BMC Genom. 16, 990 (2015).

55. Paul, A., Cai, Y., Atwal, G. S. \& Huang, Z. J. Developmental coordination of gene expression between synaptic partners during GABAergic circuit assembly in cerebellar cortex. Front. Neural Circuits 6, 37 (2012).

56. Stoodley, C. J. The cerebellum and neurodevelopmental disorders. Cerebellum 15, 34-37 (2016)

57. Enga, R. M. et al. Initial characterization of behavior and ketamine response in a mouse knockout of the post-synaptic effector gene Anks1b. Neurosci. Lett. 641, 26-32 (2017).

58. Chahrour, M. \& Zoghbi, H. Y. The story of Rett syndrome: from clinic to neurobiology. Neuron 56, 422-437 (2007).
59. Scholz, C. J. et al. Explorative results from multistep screening for potential genetic risk loci of Alzheimer's disease in the longitudinal VITA study cohort. J. Neural Transm. (Vienna) 125, 77-87 (2018).

60. Moreira-Filho, C. A. et al. Community structure analysis of transcriptional networks reveals distinct molecular pathways for early- and late-onset temporal lobe epilepsy with childhood febrile seizures. PLOS ONE 10, e0128174 (2015).

61. Croteau-Chonka, D. C. et al. Genome-wide association study of anthropometric traits and evidence of interactions with age and study year in Filipino women. Obes. (Silver Spring) 19, 1019-1027 (2011).

62. Meng, T. et al. Identification of differential gene expression profiles in placentas from preeclamptic pregnancies versus normal pregnancies by DNA microarrays. OMICS 16, 301-311 (2012).

63. Kerner-Rossi, M., Gulinello, M., Walkley, S. \& Dobrenis, K. Pathobiology of Christianson syndrome: linking disrupted endosomal-lysosomal function with intellectual disability and sensory impairments. Neurobiol. Learn. Mem. pii: S1074-7427(18)30114-X (2018).

64. Gromova, K. V. et al. Neurobeachin and the kinesin KIF21B are critical for endocytic recycling of NMDA receptors and regulate social behavior. Cell Rep. 23, 2705-2717 (2018).

65. Endele, S. et al. Mutations in GRIN2A and GRIN2B encoding regulatory subunits of NMDA receptors cause variable neurodevelopmental phenotypes. Nat. Genet. 42, 1021-1026 (2010)

66. Rumbaugh, G., Adams, J. P., Kim, J. H. \& Huganir, R. L. SynGAP regulates synaptic strength and mitogen-activated protein kinases in cultured neurons. Proc. Natl Acad. Sci. USA 103, 4344-4351 (2006).

67. Wang, X. et al. Loss of sorting nexin 27 contributes to excitatory synaptic dysfunction by modulating glutamate receptor recycling in Down's syndrome. Nat. Med. 19, 473-480 (2013).

68. Neagoe, I. et al. The GluN2B subunit represents a major functional determinant of NMDA receptors in human induced pluripotent stem cellderived cortical neurons. Stem Cell Res. 28, 105-114 (2018).

69. Zhao, X. \& Bhattacharyya, A. Human models are needed for studying human neurodevelopmental disorders. Am. J. Hum. Genet. 103, 829-857 (2018).

70. Giorgetti, A. et al. Cord blood-derived neuronal cells by ectopic expression of Sox2 and c-Myc. Proc. Natl Acad. Sci. USA 109, 12556-12561 (2012).

71. Lopez-Granero, C. et al. BXD recombinant inbred strains participate in social preference, anxiety and depression behaviors along sex-differences in cytokines and tactile allodynia. Psychoneuroendocrinology 80, 92-98 (2017).

72. Gulinello, M., Orman, R. \& Smith, S. S. Sex differences in anxiety, sensorimotor gating and expression of the alpha4 subunit of the GABAA receptor in the amygdala after progesterone withdrawal. Eur. J. Neurosci. 17, 641-648 (2003).

73. Molero, A. E. et al. Selective expression of mutant huntingtin during development recapitulates characteristic features of Huntington's disease. Proc. Natl Acad. Sci. USA 113, 5736-5741 (2016).

74. Mike, E. V. et al. Neuropsychiatric Systemic Lupus Erythematosus Is Dependent on Sphingosine-1-Phosphate Signaling. Front. Immunol. 9, 2189 (2018).

75. Gulinello, M. et al. Rigor and reproducibility in rodent behavioral research. Neurobiol. Learn Mem. pii: S1074-7427(18)30001-7 (2018).

76. Van Segbroeck, M., Knoll, A. T., Levitt, P. \& Narayanan, S. MUPET-mouse ultrasonic profile ExTraction: a signal processing tool for rapid and unsupervised analysis of ultrasonic vocalizations. Neuron 94, 465-485 e5 (2017).

77. Rappsilber, J., Mann, M. \& Ishihama, Y. Protocol for micro-purification, enrichment, pre-fractionation and storage of peptides for proteomics using StageTips. Nat. Protoc. 2, 1896-1906 (2007).

78. Erdjument-Bromage, H., Huang, F. K. \& Neubert, T. A. Sample preparation for relative quantitation of proteins using tandem mass tags (TMT) and mass spectrometry (MS). Methods Mol. Biol. 1741, 135-149 (2018).

79. Cox, J. et al. Andromeda: a peptide search engine integrated into the MaxQuant environment. J. Proteome Res 10, 1794-1805 (2011).

80. Tyanova, S., Temu, T. \& Cox, J. The MaxQuant computational platform for mass spectrometry-based shotgun proteomics. Nat. Protoc. 11, 2301-2319 (2016).

\section{Acknowledgements}

Supported by NIH R01-NIA-AG039521, NIH R56MH115201, and NIH 1UL1TR00255601 to B.A.J. and NIH T32GM007288 to A.U.C. Significant support for this work came from the Rose F. Kennedy Intellectual and Developmental Disabilities Research Center (IDDRC), which is funded through a center grant from the Eunice Kennedy Shriver National Institute of Child Health \& Human Development (NICHD U54HD090260). We acknowledge funding from the Ontario Brain Institute and the resources of MSSNG (www.mss.ng), Autism Speaks, and the Center for Applied Genomics at the Hospital for Sick Children, Toronto, Canada. This study makes use of data generated by the DECIPHER community. A full list of centers who contributed to the generation of the data is 
available from http://decipher.sanger.ac.uk and via email from decipher@sanger.ac.uk. Funding for the project was provided by the Wellcome Trust. We thank the participating families for their time and contributions to this work.

\section{Author contributions}

A.U.C. and B.A.J. designed and performed fractionation, induced neuron experiments, behavioral assays in adult mice and pups, immunoprecipitations, and interactome analysis. C.H.C. designed and performed neuronal induction from iPSCs and morphometry of mice. J.O.T. and S.C. developed Nestin-Het mouse model and performed preliminary behavioral assays. A.U.C., P.A.C., J.C.B. and S.M. designed and carried out human phenotyping. H.E.B. and T.A.N. performed isobaric labeling and mass spectrometry. A.I., E.A., S.W.S., I.K., J.R. and E.B. provided patient genotyping and phenotyping information. A.U.C., S.M. and B.A.J. interpreted results and wrote the paper.

\section{Additional information}

Supplementary Information accompanies this paper at https://doi.org/10.1038/s41467019-11437-w.

Competing interests: The authors declare no competing interests.

Reprints and permission information is available online at http://npg.nature.com/ reprintsandpermissions/
Peer review information: Nature Communications thanks Changuk Chung, and the other, anonymous, reviewer(s) for their contribution to the peer review of this work. Peer reviewer reports are available.

Publisher's note: Springer Nature remains neutral with regard to jurisdictional claims in published maps and institutional affiliations.

\section{(c) (1)}

Open Access This article is licensed under a Creative Commons Attribution 4.0 International License, which permits use, sharing, adaptation, distribution and reproduction in any medium or format, as long as you give appropriate credit to the original author(s) and the source, provide a link to the Creative Commons license, and indicate if changes were made. The images or other third party material in this article are included in the article's Creative Commons license, unless indicated otherwise in a credit line to the material. If material is not included in the article's Creative Commons license and your intended use is not permitted by statutory regulation or exceeds the permitted use, you will need to obtain permission directly from the copyright holder. To view a copy of this license, visit http://creativecommons.org/ licenses/by/4.0/.

(C) The Author(s) 2019 